OPEN ACCESS

Edited by:

Andreas Pircher,

Innsbruck Medical University, Austria

Reviewed by:

Christoph Reinhardt,

Johannes Gutenberg University Mainz,

Germany

Federica Facciotti,

European Institute of Oncology (IEO),

Italy

${ }^{*}$ Correspondence:

Xuedong Zhou

zhouxd@scu.edu.cn

Lei Cheng

chenglei@scu.edu.cn

Specialty section:

This article was submitted to

Cancer Immunity

and Immunotherapy,

a section of the journal

Frontiers in Oncology

Received: 06 June 2021 Accepted: 23 August 2021 Published: 13 September 2021

Citation:

Li B, Gong T, Hao Y, Zhou X and Cheng $L$ (2021) Mining the Gut

Microbiota for Microbial-Based

Therapeutic Strategies in

Cancer Immunotherapy.

Front. Oncol. 11:721249.

doi: 10.3389/fonc.2021.721249

\section{Mining the Gut Microbiota for Microbial-Based Therapeutic Strategies in Cancer Immunotherapy}

\author{
Bolei $\mathrm{Li}^{1,2}$, Tao Gong ${ }^{2}$, Yu Hao ${ }^{1,2}$, Xuedong Zhou ${ }^{1,2 *}$ and Lei Cheng ${ }^{1,2 *}$ \\ 1 State Key Laboratory of Oral Diseases, West China Hospital of Stomatology, National Clinical Research Center for Oral \\ Diseases, Sichuan University, Chengdu, China, ${ }^{2}$ Department of Operative Dentistry and Endodontics, West China School of \\ Stomatology, Sichuan University, Chengdu, China
}

The past two decades witnessed a revolution in our understanding of host-microbiota interactions that led to the concept of the super-organism consisting of a eukaryotic part and a prokaryotic part. Owing to the critical role of gut microbiota in modulating the host immune system, it is not beyond all expectations that more and more evidence indicated that the shift of gut microbiota influenced responses to numerous forms of cancer immunotherapy. Therapy targeting gut microbiota is becoming a promising strategy to improve cancer immunotherapy. In this review, we discuss the role of the gut microbiota in response to cancer immunotherapy, the mechanisms that the gut microbiota influences cancer immunotherapy, and therapeutic strategies targeting gut microbiota to improve cancer immunotherapy.

Keywords: gut microbiota, cancer immunotherapy, MAMPs, microbial metabolites, fecal microbiota transplant

\section{INTRODUCTION}

Over the past decades, immunotherapy has emerged as a mainstay in cancer treatment, with the advances in our understanding of cancer immunosuppressive microenvironments. Cancer immunotherapy was applied to a broad range of cancers, but $70 \%$ to $80 \%$ of patients failed to experience a life-altering durable response (1). To benefit more patients from cancer immunotherapy, efforts are made to evoke the immune response.

The gut microbiota is drawing tremendous attention given its effects on human health. Mounting evidence revealed that the gut microbiota and the immune system constantly interact $(2,3)$. Since immunotherapy was approved by US Food and Drug Administration (FDA), increasing clinical studies revealed the association between the gut microbiota and response to immunotherapy. Basing on the solid clinical association, the causal/mechanistic link of gut microbiota and immunotherapy was uncovered with preclinical models. Microbe-associated molecular patterns (MAMPs), molecular mimicry of microbial antigens with tumor neoantigen, and microbial metabolites were key factors that gut microbiota depends on to influence the response of cancer immunotherapy. Currently, more and more preclinical and clinical evidence indicated that the shift of gut microbiota influenced responses to numerous forms of cancer immunotherapy (4). As a result, therapeutic strategies targeting gut microbiota, including fecal microbiota transplant (FMT), diet, probiotics, and antibiotics, are regarded as promising candidates in improving cancer immunotherapies. Numerous clinical trials were performed to explore effective strategies to benefit cancer immunotherapy via improving gut 
microbiota. Thus, this review will mine the gut microbiota for cancer immunotherapy via summarizing and discussing the clinical-associated and causal/mechanistic links and clinical trials of gut microbiota and cancer immunotherapy, comparing the advantages and disadvantages of therapeutic strategies targeting the gut microbiota.

\subsection{Cancer Immunotherapy}

The immune system plays a dominant role in cancer control, attributed to the detection and elimination of cancer cells. On the other hand, some tumor cells escape immune surveillance by i) defecting the expression of antigen-presenting proteins, or antigen processing, or presentation, rendering them invisible to immune cell; ii) expressing proteins in inhibiting inflammation and inducing an immunosuppressive state within the tumor microenvironment; and iii) becoming insensitive to immune effector mechanisms (5). Immunotherapy helps the immune system to better act against cancer, via encouraging immune elimination and hindering immune evasion of cancer cells.

Therapeutic advances in immunotherapy have rapidly emerged in the past few years, especially the immune checkpoint inhibitors (ICIs). Currently, ICIs are FDA-approved for the treatment of many cancer types, including advanced-stage melanoma, squamous and non-squamous non-small cell lung carcinoma (NSCLC), Merkel cell carcinoma, head and neck squamous cell carcinoma, urothelial carcinoma, kidney carcinoma, microsatellite instability-high or DNA mismatch repair-deficient cancers, refractory Hodgkin lymphoma, hepatocellular carcinoma, and gastric cancer $(6,7)$. Now, ICIs are coming to neoadjuvant (presurgical) era. Clinical studies $(8-10)$ have unleashed the promise of neoadjuvant immunotherapy. More than $90 \%$ of NSCLC patients were able to undergo surgery within the planned timeframe after neoadjuvant immunotherapy (11). In addition, RNA vaccine could be another effective immunotherapy, which drives immunity by the induction of strong $\mathrm{CD}^{+}$and $\mathrm{CD}^{+}$T-cell immunity against the vaccine antigens to kill cancer cells (12).

Despite the successful application of cancer immunotherapy across a broad range of human cancers, only $20 \%$ to $30 \%$ of patients experience life-altering durable response from these therapies, which varies depending on the tumor type (1). Indeed, immunotherapy responses are heterogeneous; most patients manifest primary or secondary resistance to ICIs or even acceleration of the disease, which is called "hyperprogression" (13). Efforts are being made to identify the parameters that govern the threshold of the immunity to evoke the effective anticancer immune response, defined as the "cancer immune set-point" (14).

Numerous factors have been identified to contribute to the "cancer immune set-point" via regulating overall immune status, including tumor mutational load, cell metabolism, genomic drivers, and host-specific genetic variation $(15,16)$. Also, recent investigations highlight the effect of microbiota on the parameters that govern the effectiveness of immunotherapy $(17,18)$.

\subsection{Gut Microbiota and Immunity}

The human gastrointestinal tract harbors extremely high densities of microorganisms called the microbiota. A human being is more and more perceived as a super-organism consisting of a eukaryotic part and a prokaryotic part $(19,20)$. The gut microbiota is populated with as many as 100 trillion cells (21), whose collective gene set is approximately 100 times larger than the human gene complement $(22,23)$. Since birth, gut microbiota interacts with the host constantly throughout development. In consequence, it is not beyond all expectations that gut microbiota plays an important role in numerous host functions including immunity $(2,17)$.

In addition to influencing localized immune responses, what is more, gut microbiota contributes to systemic innate and adaptive immunity. On the one hand, the gut microbiota is a main source of MAMPs and ligands of pattern recognition receptors (PRRs). PRRs include the Toll-like receptors (TLRs), the nucleotide-binding oligomerization (NOD)-like receptors (NLRs), the RIG-I-like receptors, the C-type lectin receptors, the absent in melanoma 2 (AIM2)-like receptors, and the OASlike receptors (24), which are widely expressed innate immune cells. In addition, gut microbiota stimulates the expression of PRRs. For example, gut microbiota orchestrates TLR expression on intestinal epithelial cells (25). MAMPs systemically prime the innate immune system, enhancing killing by bone marrowderived neutrophils $(26,27)$ and increasing constitutive production of type I interferons of plasmacytoid dendritic cells (DCs) and cross-priming of DCs $(28,29)$.

On the other hand, gut microbiota-derived metabolites educate both innate and adaptive immunity. The gut microbiota metabolized the fiber, subsequently increasing the concentration of circulating short-chain fatty acids (SCFAs). SCFAs enhance the generation of macrophage and DC precursors and their phagocytic capacity (30), induce antiinflammatory regulatory $\mathrm{T}$ cells (Tregs) (31), and facilitate antibody production of B cells (32). Polysaccharide A (PSA), a zwitterionic capsular carbohydrate, induces $\mathrm{FOXP}^{+}$Treg differentiation and the production of IL-10 (33). Purine metabolite inosine advances Th1 differentiation via adenosine $2 \mathrm{~A}$ receptors $\left(\mathrm{A}_{2 \mathrm{~A}} \mathrm{R}\right)$ (34). Therefore, it is not surprising that more and more studies are revealing the associations and mechanisms between gut microbiota and cancer immunotherapy and are exploring the strategies to improve immunotherapy by taking advantage of gut microbiota.

\subsection{The Mechanisms of Gut Microbiota Modulating Immunotherapy}

Diverse studies revealed that gut microbiota plays a crucial part in cancer immunotherapy. Both of the bacteria colonized in the gut and that translocated in the tumor or lymphoid organs regulate cancer immunotherapy. The mechanism for the immune modulation of gut microbiota is being disclosed. Based on existing researches, there are three ways by which gut microbiota influence systemic cancer immunotherapy: a) evoking the innate immunity and downstream adaptive immunity by MAMPs; b) yielding an endogenous tumor vaccine by molecular mimicry of microbial antigens with tumor neoantigen; and c) stimulating tumor-infiltrating immune cell by microbial metabolites (Figure 1). 


\subsubsection{Microbe-Associated Molecular Patterns}

MAMPs, ligands of PRRs mostly expressed on innate immune cells, can act directly on local intestinal tissue cells but also penetrate beyond the mucosa, into circulation to tune immune cells in peripheral tissues (35). MAMPS can trigger at least partial activation of innate immune cells such as DCs. Furthermore, conditional antigen-presenting cells (APCs) enhanced the ability to evoke adaptive immune response and modulates cancer immunotherapy (Figure 1A).

Commensal bacteria have been identified in extragastrointestinal tissues typically considered to be sterile. Bacteria were detected in the blood (36), lymphoid organs $(37,38)$, and various tumor tissues (39, 40). Live bacteria gaining access to tumors or lymphoid organs may initiate a strong immune response by MAMPs. For example, the stimulator of interferon genes (STING) is a direct sensor of bacterial cyclic dinucleotides. Shi et al. revealed that Bifidobacterium facilitates translocation in tumor sites, where it facilitated antiCD47 immunotherapy via STING signaling, increasing crosspriming of DCs (28) (Figure 1A). Sivan et al. showed that splenic DCs isolated from mice colonized with Bifidobacterium showed superior priming of naïve $\mathrm{CD}^{+} \mathrm{T}$ cells in vitro (41).

MAMPs can traverse the mucosal barrier and enter the circulation. Stimuli capable of activating a range of TLR and NOD receptors were detected in serum from healthy individuals (42). In cancer immunotherapy, gut microbiota enhanced cancer response to the combination of $\mathrm{CpG}$ and anti-IL-10R through increasing tumor necrosis factor (TNF) production, which depends on the activation of TLR4 on tumor amyloid cells. And gavage with bacterial lipopolysaccharide (LPS), a ligand of TLR4, largely restored TNF production in tumors of antibiotic-treated mice (43). In addition, the activation of macrophages by MAMPs enhanced the phagocytic capability (44) and then primed $\mathrm{CD} 8^{+} \mathrm{T}$ cells to exhibit cytotoxic function (45) (Figure 1A).

\subsubsection{Molecular Mimicry of Microbial Antigens With Tumor Neoantigen}

The theory of "molecular mimicry" posits that T cells elicited by bacteria or viruses accidentally recognize autoantigens as they "escape" from self-tolerance-inducing mechanisms. There were some reports that had demonstrated that microbe-specific $\mathrm{CD} 4^{+}$ or $\mathrm{CD}^{+} \mathrm{T}$ lymphocytes attack normal tissues (46-48). Some data revealed a mechanistic role for $\mathrm{T}$-cell epitopes shared between bacteria and tumor cells $(37,49-53)$. Fluckiger et al. (53) found the MHC-I-binding epitopes in the tail length tape measure protein (TMP) of a prophage. Enterococcus hirae 13144 harbored the bacteriophage that improves the response to anti-PD1 via activating TMP-specific $\mathrm{H}-2 \mathrm{~Kb}$-restricted $\mathrm{CD}^{+} \mathrm{T}$ cell. In mouse models, administration of enterococci containing the bacteriophage boosted T-cell responses. In humans, the presence of the bacteriophage was associated with improved survival after PD-1

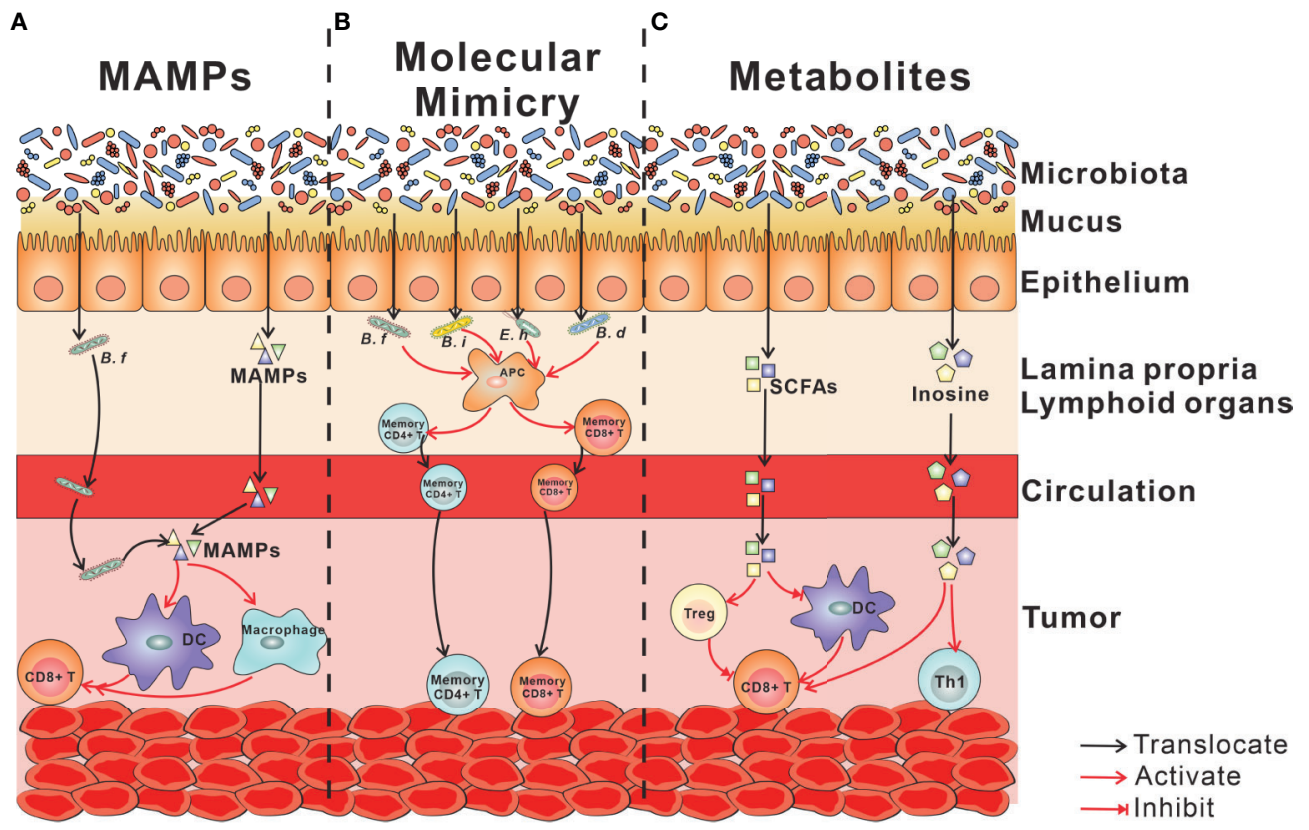

FIGURE 1 | Mechanisms linking gut microbiota with cancer immunotherapy. (A) MAMPs. Live bacteria (Bifidobacterium facilitates) and MAMPs traverse the mucosal barrier, enter the circulation, and finally locate at the tumor tissue, where MAMPs activate myeloid cells, including DCs and macrophages. The activation of myeloid cells enhances the phagocytosis of macrophages and cytotoxicity of CD8 ${ }^{+} T$ cells downstream. (B) Molecular mimicry of microbial antigens with tumor neoantigen. Antigens of commensal bacteria, including Bifidobacterium facilitate, Bifidobacterium intestinihominis, Enterococcus hirae 13144, and Bifidobacterium breve, are presented by APCs to CD4 ${ }^{+} \mathrm{T}$ cells and $\mathrm{CD} 8^{+} \mathrm{T}$ cells. By circulations, antigen-specific T cells arrive at tumor tissue and cross-react with tumor neoantigen. (C) Microbial metabolites. Microbiota-derived SCFAs play an immune-suppressive role in the tumor microenvironment via increasing the portion of Tregs, inhibiting DC maturation and CD8 ${ }^{+}$T-cell activation. Microbiota-derived inosine acts to advance Th1 differentiation and CD8 ${ }^{+} \mathrm{T}$ cytotoxicity. MAMPs, microbe-associated molecular patterns; DC, dendritic cell; APCs, antigen-presenting cells; SCFAs, short-chain fatty acids. 
immunotherapy. In addition, E. hirae and Bifidobacterium intestinihominis specific memory $\mathrm{CD}^{+} \mathrm{T}$ cells were associated with longer progression-free survival (PFS) in cancer patients (37). Memory T-cell responses against Bifidobacterium fragilis and anticancer efficacy of anti-CTLA4. Adoptive transfer of Bi. fragilisreactive $\mathrm{CD}^{+} \mathrm{T}$ cells restored anti-CTLA4 efficacy in germ-free (GF) mice (51). Bessell et al. (52) found that T cells targeting an epitope called SVYRYYGL, expressed in Bifidobacterium breve, cross-react with a model neoantigen SIYRYYGL. Compared with mice with Bifidobacterium colonization, tumors expressing the model SIYRYYGL neoantigen grew faster in mice lacking Bifidobacterium (Figure 1B).

\subsubsection{Microbial Metabolites}

Microbiota can metabolize dietary components that cannot be metabolized by the host, thus contributing to the production of primary metabolites and the modulation of secondary metabolites (54). The diverse array of metabolites in the mammalian intestine have the potential to modulate immunity. Several such microbial metabolites include SCFAs, lactic acid, spermidine, niacin, indole, retinoic acid, PSA, bile acid, and taurine (55).

SCFAs, namely, acetate, propionate, and butyrate, are the result of non-digestible carbohydrate fermentation by anaerobic commensal bacteria. In terms of immune regulation, SCFAs modulate cytokine releasing (56-58) and function of innate immune cells $(30,59,60), \mathrm{B}$ cell $(61)$, and Tregs $(31,62)$ by acting as a histone deacetylase inhibitor or ligands for G-proteincoupled receptors. In cancer immunotherapy, SCFAs play an immune-suppressive role with an increase in the abundance of Tregs (63). In the mouse model, administration of butyrate diminished the efficacy of anti-CTLA4, via inhibiting DC maturation and T-cell activation (Figure 1C). In the clinical study, cancer patients with low concentrations of SCFAs showed prolonged PFS, and an association between gut bacteria and systemic concentrations of SCFAs was found (63). However, these results are in contrast those of with two clinical studies showing that high concentrations of fecal and plasma SCFAs were associated with a response to PD-1 treatment $(64,65)$.

Although there is no evidence showing that lactic acid or spermidine from gut microbiota influence immunotherapy directly, lactic acid derived from cancer cells suppressed the function of T cells, NK cells, and macrophages, resulting in the attenuated efficiency of anti-PD-L1 and anti-CD47 $(66,67)$. Gut microbiota-derived spermidine preferentially induces naïve $\mathrm{T}$ cells to Tregs in the gut tissue (68).

The purine nucleoside inosine is generated by deamination of adenosine or the action of $5^{\prime}$-nucleotidase on inosine monophosphate. He et al. (69) revealed that gut microbiota regulated levels of the purine metabolite inosine that suppressed the differentiation and inflammation of Th1/Th2 cells via $\mathrm{A}_{2 \mathrm{~A}} \mathrm{R}$ on $\mathrm{T}$ cells. Intriguingly, Mager et al. (34) discovered that the inhibition of Th1/Th2 cells is dependent on the absence of IFN $\gamma$; when this cytokine is present, inosine acted to advance Th1 differentiation via $\mathrm{A}_{2 \mathrm{~A}} \mathrm{R}$ and boost anti-CTLA4 therapy. In addition, the translocation of inosine-producing bacteria in tumors was not required for the enhancement of immune therapy. Thus, microbiota-derived soluble inosine augments cancer immunotherapy through blood circulation. Besides signaling molecules, inosine is an essential cellular energy. Within tumors, cancer cells rapidly deplete glucose such that infiltrating $\mathrm{T}$ cells, which require abundant energy substrates for full function, would have been outcompeted if alternative substrates were not present. Wang et al. (70) demonstrated that inosine is an alternative source of energy to glucose within the tumor microenvironment; the combination of inosine supplementation and administration of anti-PD-L1 led to delayed tumor growth and increased survival time in a mouse model of melanoma. Unfortunately, some cancer cells compete with $\mathrm{T}$ cells for inosine as an energy source, which diminished the beneficial effect of inosine supplementation together with anti-PD-L1 (Figure 1C).

\section{GUT MICROBIOTA IN RESPONSE AND TOXICITY TO IMMUNOTHERAPY}

\subsection{Gut Microbiota and Immunotherapy 2.1.1 Clinical Evidence Linking Gut Microbiota and Immunotherapy}

Several clinical studies, involving Americans, Chinese, Japanese, French, and Netherlands, have demonstrated the association between gut microbiota and immunotherapy (Table 1). 16S rDNA sequencing or metagenomic shotgun sequencing (MSS) were used to analyze the composition of gut microbiota.

ICIs were first approved by the FDA to cure melanoma. The response of melanoma to ICIs was associated with a range of factors; integrative molecular and clinical modeling was used to predict the response (86). Since 2017, there have been six clinical studies, including 213 patients, that took insight into the association between the gut microbiota and the immunotherapy on metastasis melanoma $(63,71-75)$. All of them took the baseline (prior ICI treatment) microbiota into the first consideration. Totally, 26 bacteria were found by those studies to be related to a positive response in metastatic melanoma patients, including longer PFS and overall survival (OS). Among those bacteria, Faecalibacterium prausnitzii was found enriched in responders by three studies, from the United States and France, respectively (71-73). In addition, Coutzac et al. revealed that responders had increased Faecalibacterium (63). Three species of Bacteroides (72, 75), two species of Bifidobacterium (74), a species of Clostridium $(71,73)$ correlated with immunotherapy response positively. Gemmiger formicilis (71) and Gemminger (63), Ruminococcus bromii (73), and Ruminococcus (71) were reported to be enriched in responders. However, only Gopalakrishnan et al. (73) showed increased alpha diversity in responders.

The anti-PD-1/PD-L1 treatment has become the first-line strategy for NSCLC. There were four clinical studies (76-79) about gut microbiota and ICI focused on NSCLC patients, and another two $(80,81)$ included NSCLC patients. All of those studies recruited East Asian patients, except for Routy et al. (81). In addition, Katayama et al. (78) and Jin et al. (76) took the progression (during ICI treatment) microbiota into 
TABLE 1 | Clinical evidence linking gut microbiota and cancer immunotherapy.

\begin{tabular}{|c|c|c|c|c|c|}
\hline Cancer type & Therapy & $\begin{array}{l}\text { Sample } \\
\text { size }\end{array}$ & Alpha diversity & Bacteria related to response & Ref. \\
\hline Metastatic melanoma & Anti-CTLA-4 & 26 & Not mentioned & $\begin{array}{l}\text { Faecalibacterium prausnitzii, Gemmiger formicilis, butyrate-producing } \\
\text { bacteria SS2-1, Ruminococcus, Lachnospiraceae, Clostridium XIVa, } \\
\text { Blautia }\end{array}$ & (71) \\
\hline Metastatic melanoma & Anti-CTLA-4 & 38 & Not mentioned & Faecalibacterium, Gemminger & (63) \\
\hline Metastatic melanoma & $\mathrm{ICl}$ & 39 & No significant difference & $\begin{array}{l}\text { F. prausnitzii, Bacteroides thetaiotaomicron, Holdemania filiformis, } \\
\text { Bacteroides caccae }\end{array}$ & $(72)$ \\
\hline Metastatic melanoma & Anti-PD1 & 43 & Higher in responders & $\begin{array}{l}\text { F. prausnitzii, Ruminococcus bromii, Porphyromonas pasteri, Clostridium } \\
\text { hungati, Phascolarctobacterium faecium }\end{array}$ & (73) \\
\hline Metastatic melanoma & $\begin{array}{l}\text { Anti-PD1 or anti- } \\
\text { CTLA4 }\end{array}$ & 42 & Not mentioned & $\begin{array}{l}\text { Enterococcus faecium, Collinsella aerofaciens, Bifidobacterium } \\
\text { adolescentis, Klebsiella pneumoniae, Veillonella parvula, Parabacteroides } \\
\text { merdae, Lactobacillus sp., Bifidobacterium longum }\end{array}$ & $(74)$ \\
\hline Metastatic melanoma & $\mathrm{ICl}$ & 25 & No significant difference & Streptococcus parasanguinis, Bacteroides massiliensis & (75) \\
\hline NSCLC & Anti-PD1 & 25 & Higher in responders & Alistipes putredinis, B. Iongum, Prevotella copri & (76) \\
\hline NSCLC & Anti-PD1/PD-L1 & 70 & Higher in responders & Clostridiales, Ruminococcaceae UCG 13 & $(77)$ \\
\hline NSCLC & $\mathrm{ICl}$ & 17 & No significant difference & Lactobacillus, Clostridium, Syntrophococcus & (78) \\
\hline NSCLC & Anti-PD1 & 63 & No significant difference & Parabacteroides, Methanobrevibacter & (79) \\
\hline $\begin{array}{l}\text { NSCLC and gastric } \\
\text { cancer }\end{array}$ & Anti-PD1 & 38 & Higher in responders & Ruminococcaceae & (80) \\
\hline NSCLC and RCC & Anti-PD1 & 100 & Not mentioned & $\begin{array}{l}\text { Akkermansia muciniphila, Lachnospiraceae, Erysipelotrichaceae bacterium, } \\
\text { E. faecium, Alistipes indistinctus, B. caccae, Bacteroides xylanisolvens, } \\
\text { Bacteroides nordii }\end{array}$ & (81) \\
\hline $\mathrm{RCC}$ & Anti-PD1 & 22 & No significant difference & Akkermansia & $(82)$ \\
\hline Solid tumors & $\begin{array}{l}\text { Chemotherapy/ } \\
\text { immunotherapy }\end{array}$ & 26 & Higher in responders & B. xylanisolvens, Bacteroides ovatus, P. copri, Alistipes spp. & (83) \\
\hline Thoracic neoplasms & Anti-PD1 & 42 & No significant difference & $\begin{array}{l}\text { Akkermansiaceae, Enterococcaceae, Enterobacteriaceae, } \\
\text { Carnobacteriaceae, Clostridiales Family XI }\end{array}$ & (84) \\
\hline Gastric cancer & Anti-PD1 & 501 & Higher in responders & Odoribacter, Veillonella & \\
\hline Gastrointestinal cancer & Anti-PD1/PD-L1 & 74 & No significant difference & Prevotella, Ruminococcaceae, Lachnospiraceae & $(65)$ \\
\hline $\begin{array}{l}\text { Hepatocellular } \\
\text { carcinoma }\end{array}$ & Anti-PD1 & 8 & Higher in responders & $\begin{array}{l}\text { A. muciniphila, Ruminococcaceae spp., Bifidobacterium dentium, } \\
\text { Lactobacillus }\end{array}$ & (85) \\
\hline
\end{tabular}

NSCLC, non-small cell lung carcinoma; ICl, immune checkpoint inhibitor; RCC, renal cell carcinoma.

consideration. In general, a positive correlation between alpha diversity and ICI response was found in three of the six clinical studies $(76,77,80)$. Nineteen bacteria were related with a positive response, including Bacteroides (81), Bifidobacterium (76), Clostridium (78), and Ruminococcus $(78,80)$, which correlated with immunotherapy response on metastasis melanoma positively, but Faecalibacterium was not in the NSCLC list.

In addition, there are some studies that revealed the association between gut microbiota and immunotherapy in the other solid tumor models $(65,80-85)$. Three of those clinical studies found the responders with higher alpha diversity $(80,83$, 85). Worth mentioning is a clinical study involving 501 patients that also revealed the positive correlation between alpha diversity and ICI response (https://meetinglibrary.asco.org/record/ 193964/abstract). Similar to results of metastasis melanoma and NSCLC, Bacteroides (81, 83), Bifidobacterium (85), and Ruminococcus $(65,85)$ were enriched in the responders on other solid tumors. Furthermore, Akkermansia muciniphila was found enriched in responders by two individual studies (81, 85); Agarwal et al. (82) and Yin et al. (84) showed that Akkermansia correlated with beneficial response.

In summary, many clinical studies identified the association between gut microbiota and immunotherapy. Although various sample volumes from different regions, different collection techniques, cancer types, and distinctive sequencing methods limit the accuracy of gut microbial comparisons, we can find some clues from those studies. First, although only a part of the studies showed a positive correlation between alpha diversity and immunotherapy response, none of them showed a negative correlation, indicating the importance of alpha diversity. Second, Bacteroides, Bifidobacterium, Ruminococcus, and Akkermansia were frequently found to be associated with beneficial responses, indicating that they may play a role in regulating immunotherapy. Regrettably, there are no available data showing the association between gut microbiota and the efficiency of neoadjuvant immunotherapy. However, Batten et al. revealed that the diversity and composition of gut microbiota were associated with immune-related adverse events in neoadjuvant immunotherapy (87). Rajji et al. reported that antibiotics were associated with less benefit from neoadjuvant immunotherapy on bladder cancer (88).

\subsubsection{Mouse Models Showing the Effect of Gut Microbiota on Immunotherapy}

There is no available clinical trial that shows the effect of gut microbiota on immunotherapy; therefore, the effect was illustrated by mouse models only (Table 2 ).

First of all, mice with different gut microbiota show distinct responses to immunotherapy. Wild-type (WT) mice from Jackson Laboratory (JAX) and Taconic Biosciences (TAC) were reported to have a distinct gut microbiome that contributes to their distinct immune signatures (91). The JAX 
mice carrying B16 melanoma and MC38 showed enhanced response to anti-PD-L1 and anti-CD47, respectively, compared with TAC mice $(28,41)$. Besides, loss of gut microbiota by using GF mice or treating specific pathogen-free (SPF) mice with antibiotics ablated the response to immunotherapies, including anti-IL-10 receptor plus CpG-oligonucleotide on MC38 tumorbearing mice (43), anti-CTLA4 on MCA205 sarcoma-bearing mice (51), anti-CTLA4 or anti-PD1 on MC38 tumor-bearing mice $(34,89)$, anti-CD47 on MC38 tumor-bearing mice (28), and anti-PD1 on CT26 tumor-bearing mice (92). This phenomenon was a window that revealed the association of gut microbiota with immunotherapy.

Second, when given different gut microbiota, gnotobiotic mice appeared to have a distinct response to immunotherapy, which demonstrated the effect of gut microbiota on immunotherapy, as well. As mentioned above, Matson et al. (74) investigated the gut microbiota of 38 metastatic melanoma patients treated with anti-PD1 and found the difference. Reconstitution of GF mice with fecal material from responding patients could lead to improved tumor control, augmented T-cell responses, and greater efficacy of anti-PD-L1 therapy on the B16 melanoma mouse model (74). In addition, a study by Routy et al. exhibited the same benefit from responders' gut microbiota on MCA205 sarcoma (81).

Last but not least, the beneficial effects of defined bacteria on immunotherapy have also been demonstrated by mouse models. Oral supplementation with Alistipes shahii or Ruminococcus reversed immunotherapy inhibition by the antibiotic treatment, but not Lactobacillus fermentum (43). Gavage of TAC mice with Bifidobacterium species enhanced the effect of anti-PD-L1 on MC38 colon cancer, with DC activation and increased IFN $\gamma$ producing $\mathrm{CD}^{+}$ T cells (41). As well, Bifidobacterium species enhanced the effect of antiCD47 (28). CD47, known as the "don't eat me" signal, is the phagocytosis checkpoint as a new target for cancer immunotherapy (93). Bacteroides fragilis, Bacteroides thetaiotaomicron, and Burkholderia effectively aided immunotherapy of anti-CTLA4 on MCA205 sarcomas depending on intratumoral CD11 ${ }^{+}$DCs secreting IL-12 and splenic $\mathrm{ICOS}^{+} \mathrm{Ki}^{+} 7^{+} \mathrm{IFN}^{+} \mathrm{TNF}^{+} \mathrm{T}$ cells, and tumor-infiltrating $\mathrm{T}$ cells, but not Parabacteroides distasonis nor Escherichia coli nor Bacteroides uniformis (51). Oral gavage with A. muciniphila after FMT with non-responder feces restored the efficacy of anti-PD-1 on orthotopic Lewis lung carcinoma (LLC) non-small cell lung cancers and MCA205 mouse models, which depended on IL-12, with increasing recruitment of $\mathrm{CCR} 9^{+} \mathrm{CXCR} 3^{+} \mathrm{CD} 4^{+} \mathrm{T}$ cell into mouse tumor, in the mechanism (81). A similar phenotype was also revealed on renal cell carcinoma (RCC) tumor-bearing mice (94). Tanoue et al. (89) isolated 11 human gut bacteria that increased colonic IFN $\gamma^{+} \mathrm{T}$ cells, including Ruthenibacterium lactatiformans, Eubacterium limosum, Fusobacterium ulcerans, Phascolarctobacterium succinatutens, B. uniformis, Bacteroides dorei, Paraprevotella xylaniphila, P. distasonis, Parabacteroides johnsonii, Parabacteroides gordonii, and Alistipes senegalensis. Administration with the 11bacterium mix (11-mix) recovered efficacy of anti-PD1 or antiCTLA4 with infiltration with IFN $\gamma^{+} \mathrm{T}$ cells in MC38 tumor. Mager et al. (34) showed that Bifidobacterium pseudolongum, Lactobacillus johnsonii, and Olsenella species significantly enhanced efficacy of antiCTLA4 on MC38 model and azoxymethane/dextran sodium sulfate (AOM/DSS) model, with increased IFN $\gamma^{+} \mathrm{CD}^{+} \mathrm{T}$ cells and IFN $\gamma^{+}$ $\mathrm{CD}^{+} \mathrm{T}$ cells. Roberti et al. (90) found four immunogenic bacteria (B. fragilis, a non-enterotoxigenic species, Erysipelatoclostridium ramosum, and Alistipes onderdonkii), which were able to boost vaccine (oxaliplatin-exposed organoids) efficacy and anti-PD1 efficacy on $\mathrm{MC} 38$, in a $\mathrm{CD} 103^{+} \mathrm{CD} 11 \mathrm{~b}^{-} \mathrm{DC}$ [conventional type 1 DCs (cDC1)]-dependent manner.

In summary, via mouse models, the causal/mechanistic link between gut microbiota and immunotherapy was illustrated.

TABLE 2 | Gut microbiota enhancing cancer immunotherapy in mice.

\begin{tabular}{|c|c|c|c|c|}
\hline Tumor model & Therapy & Beneficial bacteria species & Specific mechanisms & Ref. \\
\hline B16 melanoma & Anti-PD-L1 & Bifidobacterium & $\mathrm{DC}, \mathrm{IFN} \gamma^{+} \mathrm{CD}^{+} \mathrm{T}$ cells & $(41)$ \\
\hline B16 SIY melanoma & Anti-PD-L1 & Responder patient FMT & $\mathrm{CD}^{+} \mathrm{T}$ cells & (74) \\
\hline RET melanoma & Anti-PD-1 & Akkermansia muciniphila, Alistipes, Enterococcus hirae & $\begin{array}{l}\mathrm{CCR}^{+} \mathrm{CXCR}^{+} \mathrm{CD}^{+} \mathrm{T} \text { cells } \\
\text { via IL-12 }\end{array}$ & $(81)$ \\
\hline MC38 colon & Anti-CD47 & Bifidobacterium & DC via STING & $(28)$ \\
\hline MC38 colon & Anti-IL-10 + CpG & Alistipes shahii, Ruminococcus & $\mathrm{TNF}^{+}$myeloid cell & (43) \\
\hline MC38 colon & Anti-CTLA4 & Bifidobacterium pseudolongum, Lactobacillus johnsonii, Olsenella species & Th1 cell, IFN $\gamma^{+} \mathrm{CD}^{+} \mathrm{T}$ cells & $(34)$ \\
\hline MC38 colon & Anti-PD-1 or Anti-CTLA4 & $\begin{array}{l}\text { Ruthenibacterium lactatiformans, Eubacterium limosum, Fusobacterium } \\
\text { ulcerans, Phascolarctobacterium succinatutens, Bacteroides uniformis, } \\
\text { Bacteroides dorei, Paraprevotella xylaniphila, Parabacteroides distasonis, } \\
\text { Parabacteroides johnsonii, Parabacteroides gordonii, and Alistipes } \\
\text { senegalensis }\end{array}$ & $\begin{array}{l}\mathrm{CD}_{103^{+}} \mathrm{DC}, \mathrm{IFN} \gamma^{+} \mathrm{CD}^{+} \mathrm{T} \\
\text { cell }\end{array}$ & $(89)$ \\
\hline MC38 colon & Anti-PD-1 & $\begin{array}{l}\text { Bacteroides fragilis, a non-enterotoxigenic species, Erysipelatoclostridium } \\
\text { ramosum, and Alistipes onderdonkii }\end{array}$ & $\mathrm{CD}_{103^{+} \mathrm{CD} 11 \mathrm{~b}^{-} \mathrm{DC}}$ & $(90)$ \\
\hline MCA205 sarcoma & Anti-CTLA4 & B. fragilis, Bacteroides thetaiotaomicron, Burkholderia & Memory T cell & $(51)$ \\
\hline MCA205 sarcoma & Anti-PD-1 & Responder patient FMT, A. muciniphila, E. hirae, Alistipes & $\begin{array}{l}\mathrm{CCR}^{+} \mathrm{CXCR} 3^{+} \mathrm{CD}^{+} \mathrm{T} \text { cells } \\
\text { via IL-12 }\end{array}$ & $(81)$ \\
\hline RENCA RCC & Anti-PD-1 + anti-CTLA4 & Responder patient FMT & $\begin{array}{l}\mathrm{CCR}^{+} \mathrm{CXCR}^{+} \mathrm{CD}^{+} \mathrm{T} \text { cells } \\
\text { via IL-12 }\end{array}$ & $(81)$ \\
\hline LLC lung carcinoma & Anti-PD-1 & A. muciniphila, Alistipes, E. hirae & $\begin{array}{l}\mathrm{CCR}^{+} \mathrm{CXCR}^{+} \mathrm{CD}^{+} \mathrm{T} \text { cells } \\
\text { via IL-12 }\end{array}$ & $(81)$ \\
\hline
\end{tabular}

RCC, renal cell carcinoma; DC, dendritic cell; FMT, fecal microbiota transplant; STING, stimulator of interferon genes; LLC, Lewis Iung carcinoma. 
Bacteroides, Bifidobacterium, Ruminococcus, Lactobacillus, Enterococcus, and Akkermansia, associated with the response to immunotherapy in clinical studies, were revealed to activate immunity and boost the efficiency of immunotherapy in mouse models.

\subsection{Gut Microbiota and Immune Response in Chemotherapy}

Although not traditionally considered as immunotherapy, effective chemotherapy is also dependent on intact immune responses; therefore, the effect of gut microbiota on conventional chemotherapy depends on modulating the immune response.

Cyclophosphamide, a prominent alkylating anticancer agent, inhibits tumor outgrowth by inducing immunogenic cancer cell death $(95,96)$, reverting immunosuppressive T cells $(97)$, and promoting Th1 and Th17 cells (98). GF or antibiotic-treated mice carrying MCA205 sarcoma lost cyclophosphamide tumor inhibition, suggesting that the gut microbiota plays a critical role in controlling cancer during cyclophosphamide treatment. While oral gavage with E. hirae clone 13144 and Barnesiella intestinihominis reinstated cyclophosphamide efficacy, but not P. distasonis, Lactobacillus plantarum, Lactobacillus reuteri, and L. johnsonii, which segmented filamentous bacteria, even other $E$. hirae isolates $(37,38,53)$. In mechanism, E. hirae clone 13144 translocated into secondary lymphoid organs, where they stimulated the generation of a specific subset of "pathogenic" Th17 cells and memory Th1 immune responses, which crossreact with tumor-associated antigens. Finally, E. hirae clone 13144 increased the intratumoral $\mathrm{CD}^{+} /$Treg ratio and enhanced chemotherapy $(37,38,53)$. Ba. intestinihominis raised chemotherapy of cyclophosphamide through yielding tumor IFN $\gamma$ T-cell infiltration (37).

\section{STRATEGIES TO IMPROVE GUT MICROBIOTA IN CANCER IMMUNOTHERAPY}

\subsection{Fecal Microbiota Transplant}

FMT is when stool from a healthy donor is made into a liquid mixture and transferred into the gut of a different person to try to reintroduce or boost helpful organisms, which represents the most direct means to manipulate the gut microbiota. Based on results from preclinical studies discussed above, FMT is considered as an intervention to treat patients undergoing immunotherapy, especially those administered with ICIs, aiming for the safety and response of the combo of FMT and immunotherapy. Currently, melanoma, prostate cancer, gastrointestinal system cancer, NSCLC, and mesothelioma are enrolled by several FMT-related clinical trials (Table 3).

The key factor of those clinical trials is the criteria of the donor. Six of nine clinical trials treated patients who respond to immunotherapy as donors (NCT04264975, NCT04116775, NCT04521075, NCT03353402, NCT04577729, and NCT03341143). Recently, the result of the phase 1 clinical trial
(NCT03353402) was published (99). To assess the safety and feasibility of fecal FMT and re-induction of anti-PD-1 immunotherapy, the trial recruited 10 patients with anti-PD-1refractory metastatic melanoma. Two FMT donors were included in the trials who had previously been treated with anti-PD-1 monotherapy and achieved a complete response. First of all, the gut microbiota of all recipients significantly differed from their baseline and closed to the donors. In detail, patients who received donor \#1 sample had a greater relative abundance of Ruminococcus and Bifidobacterium adolescentis, whereas those who received donor \#2 sample had an overrepresentation of Clostridiaceae (99). In addition, treatment increased multiple immune-related gene sets in the tumor tissue of donor \#1 group, including IFN $\gamma$-mediated signaling pathway, T-cell activation, MHC-II protein complex, DC differentiation, and Th1-type immune response (99). Most importantly, three of 10 recipients achieved objective responses, all of them from donor \#1 group, and only one recipient had a mild temporary bloating considered as an FMT-related adverse event (99). Another phase 2 clinical trial (NCT03341143) showed that six of 15 PD-1refractory patients with melanoma benefited from the FMT (100). In this study, seven donors were included, including four with a complete response and three with a partial response. Responders' recipient microbiota exhibited a significant shift toward the donor composition compared with the non-responders'. Successful FMT was enriched in Ruminococcaceae, Bifidobacteriaceae, and Lachnospiraceae. A coinciding immune activity after the FMT was found in blood and tumor microenvironment (100). Three of nine clinical trials treated healthy people as donors (NCT04056026, NCT03772899, and NCT04130763). Interestingly, the activation of immune response was also found in advanced or metastatic melanoma patients with FMT from healthy donors (NCT03772899) (101). Most importantly, these three published trials showed a favorable safety profile and represented the first clinical evidence that the gut microbiota may have an impact on antitumor immunity and potentially even responses to immunotherapies.

Besides the criteria of donors, those clinical trials differed on the FMT preparations (Table 3). Generally, FMT preparations can be performed via oral administration of lyophilized or frozen pills and capsules, or direct delivery by endoscopy. The lower routes of administration (colonoscopy or enema) appeared to be more successful than the upper routes (gastroscopy, or nasogastric and nasointestinal tubes) (102). Maybe this is the reason that most of those clinical trials administer FMT with colonoscopy.

However, to translate FMT into the clinic, there are a number of problems that we need to face. First of all is the safety issue. FDA has reported safety alerts after the death of patients receiving FMT for Clostridium difficile infection who developed infections caused by enteropathogenic bacteria contained in the FMT. Besides harmful bacteria, the harmful virus should also be screened before FMT, considering the intestinal epithelium is a tropism of SARS-CoV-2. Second issue is how to define the optimal donors. Several investigators recruit donors from patients who previously responded to immunotherapy, while 


\begin{tabular}{|c|c|c|c|c|}
\hline Interventions & Trial number & Conditions & Major microbiota and immune related outcomes & Phases \\
\hline \multicolumn{5}{|l|}{ FMT } \\
\hline FMT via colonoscopy & NCT04264975 & Solid carcinoma & Response to immunotherapy plus FMT & Not applicable \\
\hline FMT via colonoscopy & NCT04056026 & Mesothelioma & Response to Keytruda plus FMT & Early Phase 1 \\
\hline FMT via colonoscopy & NCT03772899 & Melanoma & Response to immunotherapy plus FMT & Phase 1 \\
\hline FMT via endoscopy & NCT04116775 & Metastatic castration-resistant prostate cancer & Response to pembrolizumab plus FMT & Phase 2 \\
\hline FMT via oral capsule & NCT04130763 & Gastrointestinal system cancer & Response to anti-PD-1 plus FMT & Phase 1 \\
\hline FMT via oral capsule & NCT04521075 & Metastatic melanoma or NSCLC & Response to nivolumab plus FMT & Phase 1/Phase 2 \\
\hline $\begin{array}{l}\text { FMT via colonoscopy and } \\
\text { oral capsule }\end{array}$ & NCT03353402 & Melanoma Stage IV and unresectable Stage III & Response to immunotherapy plus FMT & Phase 1 \\
\hline FMT & NCT04577729 & Melanoma Stage III and IV & Response to checkpoint inhibitor plus FMT & Not applicable \\
\hline FMT via colonoscopy & NCT03341143 & PD-1 resistant/refractory melanoma & Response to checkpoint inhibitor plus FMT & Phase 2 \\
\hline \multicolumn{5}{|l|}{ Diet } \\
\hline Fasting mimicking diet & NCT03454282 & Breast cancer or melanoma & Tumor-infiltrating lymphocytes, gut microbiota composition & Not applicable \\
\hline $\begin{array}{l}\text { Dietary supplement: } \\
\text { IGEN0206 }\end{array}$ & NCT04009122 & Non-small cell lung cancer metastatic & $\begin{array}{l}\text { Quality of life, changes in the microbiota, interleukin levels, } \\
\text { cytokines levels }\end{array}$ & Not applicable \\
\hline \multicolumn{5}{|l|}{ Probiotics } \\
\hline \multicolumn{5}{|l|}{ Probiotic Formula } \\
\hline Oral MRx0518 & NCT04193904 & Pancreatic cancer & Tumor infiltrating lymphocytes & Phase 1 \\
\hline 5 & NCT03817125 & Metastatic melanoma & Response to checkpoint inhibitor & Phase 1 \\
\hline Oral MET-4 & NCT03838601 & Head and neck squamous cell carcinoma & $\begin{array}{l}\text { Bacterial composition and diversity, blood immune cell } \\
\text { profiling }\end{array}$ & not applicable \\
\hline Oral BB536, LA1 & NCT00936572 & Colorectal cancer & Immune and inflammatory response, bacterial translocation & Phase 2 \\
\hline IV JNJ-64041809 & NCT02625857 & Metastatic castration-resistant prostate cancer & Immune responses & Phase 1 \\
\hline Oral MR×0518 & NCT03637803 & Solid tumors & $\begin{array}{l}\text { Clinical benefit of MRx0518 in combination with } \\
\text { pembrolizumab }\end{array}$ & Phase 1/Phase 2 \\
\hline Oral RBX7455 & NCT04139993 & Breast cancer & Intratumoral immunomodulatory & Early Phase 1 \\
\hline Oral GEN-001 & NCT04601402 & Solid tumors & Response to avelumab & Phase 1 \\
\hline Oral EDP1503 & NCT03595683 & Melanoma & Response to pembrolizumab & Phase 2 \\
\hline Oral MET-4 & NCT03686202 & Solid tumors & $\begin{array}{l}\text { Relative abundance of immunotherapy-responsiveness } \\
\text { associated species of MET- } 4\end{array}$ & Early Phase 1 \\
\hline Oral VE800 & NCT04208958 & Selected types of advanced or metastatic cancer & Safety and efficacy of VE800 in combination with nivolumab & Phase 1/Phase 2 \\
\hline Oral MRx0518 & NCT03934827 & Solid tumors & $\begin{array}{l}\text { Safety, tolerability, and immune system modulation of } \\
\text { MRx0518 }\end{array}$ & Phase 1 \\
\hline Oral EDP1503 & NCT03775850 & Colorectal cancer, breast cancer, and checkpoint inhibitor relapsed tumors & $\begin{array}{l}\text { Safety, tolerability, and efficacy of EDP1503 alone and in } \\
\text { combination with pembrolizumab }\end{array}$ & Phase 1/Phase 2 \\
\hline \multicolumn{5}{|c|}{ e } \\
\hline Oral vancomycin & NCT03785210 & $\begin{array}{l}\text { Refractory primary hepatocellular carcinoma or liver-dominant metastatic cancer } \\
\text { from colorectal or pancreatic cancers }\end{array}$ & Response to nivolumab & Phase 2 \\
\hline
\end{tabular}

FMT, fecal microbiota transplant; NSCLC, non-small cell lung carcinoma. 
others prefer healthy volunteers. Now only three positive results have been published. Two of them showed the benefits from responding patients, and one of them showed the benefits from healthy people. Considering that most studies revealed the difference of gut microbiota between responders and nonresponders, it seems that responding patients should be better donors. In addition, the kinds of pathologies of the donor should be excluded. In one case, the obese phenotype has been transferred from a donor to a recipient (103). Last, FMT may benefit from host conditioning, including diet, probiotics, and antibiotics. Further studies are needed to make a synergetic combo of the FMT and host conditioning.

\subsection{Diet}

As a dominant determinant of interindividual microbiota variation $(104,105)$, diet is the key determinant of the microbiota configuration, through modulation of the abundance of microbial species and their individual or collective functions (106-108). Hippocrates noted "Let food be thy medicine and medicine be thy food." Owing to the advantageous safety, cost, and availability, diet could be a promising clinical intervention to modulate gut microbiota and downstream immune in cancer patient populations.

Prebiotics are a source of diet for your gut's healthy bacteria. They are carbs that our body cannot digest. The well-known prebiotics, microbiota-accessible carbohydrates, have a major impact on gut microbiota composition, diversity, and richness (109). Microbiota-accessible carbohydrates are fermented by gut microbiota to produce SCFAs, which have been discussed above in modulating immunotherapy. It benefited the exclusion of pathogens such as Citrobacter rodentium and $C$. difficile $(110,111)$. Another prebiotic, plant polysaccharide inulin, increased both Faecalibacterium and Bifidobacterium species in gut microbiota, which are considered potentially favorable for immunotherapy (112). The effect of a dietary supplemental nutritional product (IGEN0206) on the quality of life, nutritional status, and shift in the gut microbiota of patients with NSCLC was investigated by a clinical study (NCT04552418). Unfortunately, we have no idea of the prebiotics in IGEN0206.

Besides prebiotics, the main components of diets shift gut microbiota and immunity, including calorie, protein, and fat. A plant-based, calorie-restricted, low-protein diet, also known as fasting mimicking diet (FMD), modulated gut microbiota composition and immune cell profiles to reduce inflammatory bowel disease pathology (113). It has been proposed as a potential anticancer dietary intervention by enhancing cytotoxic $\mathrm{CD}^{+}$tumor-infiltrating lymphocytes (114). At present, NCT03454282 is designed to explore the impact of FMD on the gut microbiota composition, peripheral blood mononuclear cells, tumor-infiltrating lymphocytes, and metabolic parameters of breast cancer or melanoma patients.

The population structure responds to acute dietary change, as evidenced by rapid and substantial increases in populations at the genus and species levels. However, dietary change does not necessarily induce a permanent compositional shift, at least at the phylum level, although evidence for this assertion is limited (115). As a result, diets might not able to reshape the gut microbiota as dramatically as FMT. But the advantage in safety and convenience of diets is obvious. Considering the restricted effect of diets on gut microbiota, the combination of diets and FMT might give their advantages a full play to modulate gut microbiota and immunotherapy.

\subsection{Probiotics}

Beneficial or immune-modulating bacteria could be administered as a probiotic to manipulate cancer immunotherapy. Probiotics could provide a more feasible method of microbial manipulation in the clinical setting. Many clinical trials using probiotics in cancer patients have been initiated with some completed (Table 3).

Most of the probiotics are composed of single strains. MRx0518 is a strain of Enterococcus gallinarum, isolated from a healthy human fecal sample (116). EDP1503 is a strain of Bifidobacterium animalis subsp. lactis, BB536 is a strain of Bifidobacterium longum, and LA1 is a strain of Lactobacillus acidophilus. JNJ-64041809 is a live attenuated, double-deleted Listeria administered intravenously. GEN-001 is a single-strain bacteria isolated from the gut of healthy human volunteers. As mentioned above, Enterococcus, Bifidobacterium, and Lactobacillus are related to immunotherapy $(74,76,81,85)$. Especially, Bifidobacterium species have been demonstrated to enhance the response to ICIs in animal models by several studies $(28,34,41)$. Currently, initial data from the first six patients of NCT03637803 showed that MRx0518 combined with pembrolizumab is well tolerated in patients with solid tumors who have developed resistance to anti-PD-1/PD-L1. Two patients have shown a partial response with evidence of increased tumorinfiltrating lymphocytes, according to the RECIST v1.1 criteria 1. One additional patient has a stable disease. No drug-related serious adverse events have been noted (https://www.londonstockexchange. com/news-article/DDDD/clinical-observations-from-mrx0518/ 14295955). Initial data of NCT03775850 show that an overall response rate (ORR) of $25 \%(2 / 8)$ and a disease control rate of $37.5 \%(3 / 8)$ were observed across all triple negative breast cancer (TNBC) subjects receiving high-dose EDP1503. ORR was 33\% (2/6) among response-evaluable patients on the high dose, with two patients awaiting first response assessment. Historic studies of antiPD-1 monotherapy in heavily pretreated TNBC patients have yielded an ORR of 5\%-10\% (https://ir.evelobio.com/news-releases/newsrelease-details/evelo-biosciences-present-clinical-data-phase-12trial-edp1503). NCT02625857 showed that JNJ-64041809 has a manageable safety profile and activation of the immune response. Nevertheless, observed immune activation with monotherapy did not translate into clinical activity (117).

The probiotics could also be a consortium of live bacteria, including Primal Defense Ultra Probiotic Formula, SER-401, and MET-4 and VE800. VE800, which consisted of 11 clonal human commensal bacteria strains, activated the immunotherapy via $\mathrm{CD}^{+} \mathrm{T}$ cells in animal models (89). The consortium seems more powerful in shifting gut microbiota than single bacteria; however, it is a pity that there are no available clinical data to show the safety of probiotic consortiums, although five clinical trials are going to reveal the safety and clinical response.

In addition to the strain isolated from humans, synthetically engineered microorganisms can also be implanted as probiotics. 
Advances in synthetic biology are enabling the design of microorganisms based on therapeutic needs. Currently, engineered a non-pathogenic E. coli strain, expressing encoded nanobody antagonist of CD47, or nanobodies targeting PD-L1 and CTLA4, or STING agonist, were administered to activate systemic antitumor immunity and to regress tumor burden in mouse models (118-120), although, until now, those engineered probiotics were designed to kill tumors, directly. Along with a deep understanding of the role of gut microbiota in cancer immunotherapy, engineered probiotics will be applied to modulating gut microbiota, as an adjuvant of immunotherapy.

We believe that probiotics are the future to improve gut microbiota for immunotherapy. Compared with FMT, probiotics do not need donors nor the criteria for donors. In addition, probiotics contain less harmful and dispensable matter. Last, probiotics are easier for the industry. However, a deeper understanding of the mechanism between gut microbiota and immunotherapy is needed to develop immunotherapeutic probiotics.

\subsection{Antibiotics}

Antibiotic administration is another straightforward intervention to module gut microbiota and the downstream cancer immunotherapies. By removing harmful bacteria, some antibiotics can provide a positive effect on the gut microbiota and immunotherapy. Vancomycin targets gram-positive bacteria, including butyrate-producing bacteria and decreasing SCFA concentrations. Vancomycin treatment induced an increase of systemic $\mathrm{CD} 8 \alpha^{+} \mathrm{DCs}$, tumor-associated antigen cross-priming with antitumor $\mathrm{CD}^{+} \mathrm{T}$ cell elicitation, and tumor growth inhibition in mice, via decreasing SCFAs $(121,122)$. Recently, a phase 2 single-arm clinical trial (NCT03785210) was designed to investigate if nivolumab given with tadalafil and vancomycin causes liver tumor to shrink (Table 3).

Nevertheless, antibiotic classes should be carefully considered. Due to the lack of specificity, antibiotics decrease bacterial diversity, eliminate beneficial bacteria, and give rise to dysbiosis. As a matter of fact, numerous clinical studies from France, China, Japan, Canada, and the United States unleashed antibiotic treatment prior to immunotherapy was associated with reduced clinical benefit on melanoma (123), NSCLC $(81,124-127)$, and RCC (81, $94,124,128)$. All of those studies found that patients with antibiotic treatment prior to immunotherapy had decreased diversity of gut microbiota and worse PFS and OS. To some extent, the results are consistent with the investigation of responders and non-responders showing a positive correlation between alpha diversity and immunotherapy response. On the other hand, taking the advantage of broad-spectrum depletion of naïve gut microbiota, antibiotics could be used before FMT to achieve better microbial modulation. For instance, Baruch et al. (99) treated patients with vancomycin and neomycin to deplete their own native microbiota before FMT via colonoscopy and via oral capsules.

\section{DISCUSSION}

The dynamic nature of the microbiota makes it an attractive target for therapeutic intervention in a range of conditions, as engraftment or elimination of particular microorganisms. The shift of gut microbiota contributes to altering both innate and adaptive immunity. In addition, many studies incorporating preclinical and clinical studies have gained our insight into the influence of gut microbiota on cancer immunotherapy. Via MAMPs, microbial metabolites, and molecular mimicry, the gut microbiota educates both local and systematic immunity to alter the response to cancer immunotherapy. Therefore, in the age of microbiome, therapeutic strategies targeting gut microbiota, including FMT, diet, probiotics, and antibiotics, are developed to enhance responses to cancer immunotherapy. However, there is still a great deal to investigate the inherent mechanisms, as well as optimal strategies.

To identify causal host-microbiota relationships and mechanisms, there are two approaches generally, the microbiota-based approach and the molecule-based approach (129). The microbiota-based approach is the more often used. First, a complex microbiota is found to promote a given phenotype. Then several methods, including 16S DNA sequence, antibiotic treatment, and in vitro culture, are used to narrow down the entire microbiota to a single effector species or consortium. Furthermore, single species intervention and/or bacterial genetic engineering studies are performed to uncover the mechanisms. The molecule-based approach starts from a small molecule, which is proven to promote a given phenotype. Then by searching genomic databases or the literature, the biosynthetic machinery of the molecules and the functional species will be identified. Because many metabolic pathways are conserved in bacteria, the molecule-based approach bacteria may be possible to identify several effector species. If necessary, further investigations are needed to identify the most critical species. Up to now, nearly all of the mechanism studies focusing on the role of gut microbiota in response to immunotherapy belong to microbiota-based approaches (28, $34,37,41,43)$. No study used a molecule-based approach to explore the host-microbiota relationships in cancer immunotherapy. Given the fact that there are many sensitive in vitro models in investigating cancer immunotherapy (130, 131), systematic screening of microbiota-derived molecules with those models is an effective method to identify the molecules associated with the given phenotype. Furthermore, the systematic screen will provide one or more great starts for the molecule-based approach in further revealing the inherent mechanisms. Therefore, the molecule-based approach should be a window to explore causal host-microbiota relationships and mechanisms in cancer immunotherapy.

Furthermore, additional complexities exist as we move forward with optimal microbiota-based strategies to improve therapeutic responses. First, although these clinical studies drew similar conclusions those clinical studies linking gut microbiota and immunotherapy and some beneficial bacteria have been identified by clinical and preclinical studies, there were diverse results; it is not very clear what composition of the gut microbiome is optimal to facilitate antitumor immunity. More researches should be performed to define the ideal gut microbiota for immunotherapy. Second, although there are various range of therapeutic options to 


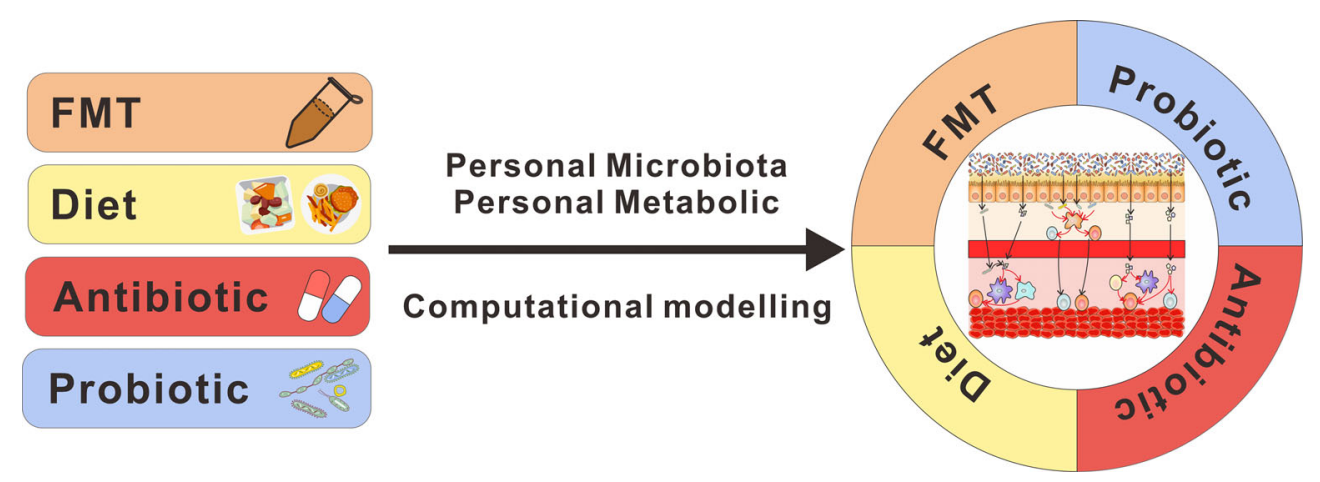

FIGURE 2 | Design of microbial therapeutics to benefit cancer immunotherapy. Stable microbial engraftment is manipulated by intrinsic microbiota, extrinsic nutrients, and colonic metabolic. As a tool, computational modeling can be used to predict the engraftment of microbiota members. Thus, with the deeper insight into this field, the rational design of microbial therapeutics will take all of those factors into consideration and combine those individual therapeutic strategies to improve cancer immunotherapy.

shift gut microbiota, precise modulation with gut microbiota remains difficult owing to the interindividual heterogeneity inherent in humans. Computational models could help in the precise design of microbial therapeutics, which can be used to predict the engraftment of immunomodulatory microbiota members (132). Based on taxonomic analysis of gut microbiota, machine learning can provide new insights to predict disease states and outcomes, which is beneficial for personalized medicine (133). Last, stable microbial engraftment can be manipulated by intrinsic microbiota, extrinsic nutrients $(134,135)$, colonic metabolic state (136), and immune state (137). Thus, precision medicine interventions in gut microbiota and a rational combo of those individual therapeutic strategies are required to optimize to match the genetic, microbial, and metabolic profiles (Figure 2). Unfortunately, we lack the ability to reliably predict how these factors influence bacteria and their immunomodulatory properties,

\section{REFERENCES}

1. Duffy MJ, Crown J. Biomarkers for Predicting Response to Immunotherapy With Immune Checkpoint Inhibitors in Cancer Patients. Clin Chem (2019) 65:1228-38. doi: 10.1373/clinchem.2019.303644

2. Ost KS, Round JL. Communication Between the Microbiota and Mammalian Immunity. Annu Rev Microbiol (2018) 72:399-422. doi: 10.1146/annurev-micro-090817-062307

3. Thaiss CA, Zmora N, Levy M, Elinav E. The Microbiome and Innate Immunity. Nature (2016) 535:65-74. doi: 10.1038/nature18847

4. Fessler J, Matson V, Gajewski TF. Exploring the Emerging Role of the Microbiome in Cancer Immunotherapy. J Immunother Cancer (2019) 7:108. doi: 10.1186/s40425-019-0574-4

5. Schreiber RD, Old LJ, Smyth MJ. Cancer Immunoediting: Integrating Immunity's Roles in Cancer Suppression and Promotion. Sci (New York NY) (2011) 331:1565-70. doi: 10.1126/science.1203486

6. Sharma P, Allison JP. The Future of Immune Checkpoint Therapy. Sci (New York NY) (2015) 348:56-61. doi: 10.1126/science.aaa8172

7. Zappasodi R, Merghoub T, Wolchok JD. Emerging Concepts for Immune Checkpoint Blockade-Based Combination Therapies. Cancer Cell (2018) 33:581-98. doi: 10.1016/j.ccell.2018.03.005

8. Blank CU, Rozeman EA, Fanchi LF, Sikorska K, van de Wiel B, Kvistborg P, et al. Neoadjuvant Versus Adjuvant Ipilimumab Plus Nivolumab in currently. Although the promise of microbial therapy has been revealed in cancer immunotherapy, a number of further studies are still needed to optimize therapeutic strategies.

\section{AUTHOR CONTRIBUTIONS}

$\mathrm{BL}, \mathrm{TG}$, and $\mathrm{YH}$ wrote the paper. $\mathrm{XZ}$ and $\mathrm{LC}$ revised the paper. All authors contributed to the article and approved the submitted version.

\section{FUNDING}

This study was supported by the National Natural Science Foundation of China 81870759 (LC) and 82071106 (LC).
Macroscopic Stage III Melanoma. Nat Med (2018) 24:1655-61. doi: 10.1038/s41591-018-0198-0

9. Huang AC, Orlowski RJ, Xu X, Mick R, George SM, Yan PK, et al. A Single Dose of Neoadjuvant PD-1 Blockade Predicts Clinical Outcomes in Resectable Melanoma. Nat Med (2019) 25:454-61. doi: 10.1038/s41591019-0357-y

10. Rozeman EA, Menzies AM, van Akkooi ACJ, Adhikari C, Bierman C, van de Wiel BA, et al. Identification of the Optimal Combination Dosing Schedule of Neoadjuvant Ipilimumab Plus Nivolumab in Macroscopic Stage III Melanoma (OpACIN-Neo): A Multicentre, Phase 2, Randomised, Controlled Trial. Lancet Oncol (2019) 20:948-60. doi: 10.1016/S1470-2045 (19)30151-2

11. Ulas EB, Dickhoff C, Schneiders FL, Senan S, Bahce I. Neoadjuvant Immune Checkpoint Inhibitors in Resectable Non-Small-Cell Lung Cancer: A Systematic Review. ESMO Open (2021) 6:100244. doi: 10.1016/ j.esmoop.2021.100244

12. Sahin U, Oehm P, Derhovanessian E, Jabulowsky RA, Vormehr M, Gold M, et al. An RNA Vaccine Drives Immunity in Checkpoint-Inhibitor-Treated Melanoma. Nature (2020) 585:107-12. doi: 10.1038/s41586-020-2537-9

13. Champiat S, Ferrara R, Massard C, Besse B, Marabelle A, Soria JC, et al. Hyperprogressive Disease: Recognizing a Novel Pattern to Improve Patient Management. Nat Rev Clin Oncol (2018) 15:748-62. doi: 10.1038/s41571018-0111-2 
14. Chen DS, Mellman I. Elements of Cancer Immunity and the CancerImmune Set Point. Nature (2017) 541:321-30. doi: 10.1038/nature21349

15. Tran L, Theodorescu D. Determinants of Resistance to Checkpoint Inhibitors. Int J Mol Sci (2020) 21:1594. doi: 10.3390/ijms21051594

16. Kishton RJ, Sukumar M, Restifo NP. Metabolic Regulation of T Cell Longevity and Function in Tumor Immunotherapy. Cell Metab (2017) 26:94-109. doi: 10.1016/j.cmet.2017.06.016

17. Gopalakrishnan V, Helmink BA, Spencer CN, Reuben A, Wargo JA. The Influence of the Gut Microbiome on Cancer, Immunity, and Cancer Immunotherapy. Cancer Cell (2018) 33:570-80. doi: 10.1016/j.ccell. 2018.03.015

18. Frankel AE, Deshmukh S, Reddy A, Lightcap J, Hayes M, McClellan S, et al. Cancer Immune Checkpoint Inhibitor Therapy and the Gut Microbiota. Integr Cancer Ther (2019) 18:1534735419846379. doi: 10.1177/1534735419846379

19. Thaiss CA, Levy M, Suez J, Elinav E. The Interplay Between the Innate Immune System and the Microbiota. Curr Opin Immunol (2014) 26::41-48. doi: 10.1016/j.coi.2013.10.016

20. Esser D, Lange J, Marinos G, Sieber M, Best L, Prasse D, et al. Functions of the Microbiota for the Physiology of Animal Metaorganisms. J Innate Immun (2019) 11:393-404. doi: 10.1159/000495115

21. Ley RE, Peterson DA, Gordon JI. Ecological and Evolutionary Forces Shaping Microbial Diversity in the Human Intestine. Cell (2006) 124:83748. doi: 10.1016/j.cell.2006.02.017

22. Qin J, Li R, Raes J, Arumugam M, Burgdorf KS, Manichanh C, et al. A Human Gut Microbial Gene Catalogue Established by Metagenomic Sequencing. Nature (2010) 464:59-65. doi: 10.1038/nature08821

23. Levy M, Thaiss CA, Elinav E. Metagenomic Cross-Talk: The Regulatory Interplay Between Immunogenomics and the Microbiome. Genome Med (2015) 7:120. doi: 10.1186/s13073-015-0249-9

24. Thaiss CA, Levy M, Itav S, Elinav E. Integration of Innate Immune Signaling. Trends Immunol (2016) 37:84-101. doi: 10.1016/j.it.2015.12.003

25. Hormann N, Brandao I, Jackel S, Ens N, Lillich M, Walter U, et al. Gut Microbial Colonization Orchestrates TLR2 Expression, Signaling and Epithelial Proliferation in the Small Intestinal Mucosa. PloS One (2014) 9: e113080. doi: 10.1371/journal.pone.0113080

26. Zhang D, Chen G, Manwani D, Mortha A, Xu C, Faith JJ, et al. Neutrophil Ageing is Regulated by the Microbiome. Nature (2015) 525:528-32. doi: 10.1038 /nature 15367

27. Clarke TB, Davis KM, Lysenko ES, Zhou AY, Yu Y, Weiser JN. Recognition of Peptidoglycan From the Microbiota by Nod1 Enhances Systemic Innate Immunity. Nat Med (2010) 16:228-31. doi: 10.1038/nm.2087

28. Shi $\mathrm{Y}$, Zheng $\mathrm{W}$, Yang $\mathrm{K}$, Harris KG, Ni K, Xue L, et al. Intratumoral Accumulation of Gut Microbiota Facilitates CD47-Based Immunotherapy via STING Signaling. J Exp Med (2020) 217:e20192282. doi: 10.1084/ jem. 20192282

29. Schaupp L, Muth S, Rogell L, Kofoed-Branzk M, Melchior F, Lienenklaus S, et al. Microbiota-Induced Type I Interferons Instruct a Poised Basal State of Dendritic Cells. Cell (2020) 181:1080-96.e1019. doi: 10.1016/j.cell.2020.04.022

30. Trompette A, Gollwitzer ES, Yadava K, Sichelstiel AK, Sprenger N, NgomBru C, et al. Gut Microbiota Metabolism of Dietary Fiber Influences Allergic Airway Disease and Hematopoiesis. Nat Med (2014) 20:159-66. doi: $10.1038 / \mathrm{nm} .3444$

31. Arpaia N, Campbell C, Fan X, Dikiy S, van der Veeken J, deRoos P, et al. Metabolites Produced by Commensal Bacteria Promote Peripheral Regulatory T-Cell Generation. Nature (2013) 504:451-5. doi: 10.1038/ nature 12726

32. Kim M, Qie Y, Park J, Kim CH. Gut Microbial Metabolites Fuel Host Antibody Responses. Cell Host Microbe (2016) 20:202-14. doi: 10.1016/ j.chom.2016.07.001

33. Mazmanian SK, Liu CH, Tzianabos AO, Kasper DL. An Immunomodulatory Molecule of Symbiotic Bacteria Directs Maturation of the Host Immune System. Cell (2005) 122:107-18. doi: 10.1016/j.cell.2005.05.007

34. Mager LF, Burkhard R, Pett N, Cooke NCA, Brown K, Ramay H, et al. Microbiome-Derived Inosine Modulates Response to Checkpoint Inhibitor Immunotherapy. Sci (New York NY) (2020) 369:1481-9. doi: 10.1126/ science.abc3421

35. Belkaid Y, Harrison OJ. Homeostatic Immunity and the Microbiota. Immunity (2017) 46:562-76. doi: 10.1016/j.immuni.2017.04.008
36. Poore GD, Kopylova E, Zhu Q, Carpenter C, Fraraccio S, Wandro S, et al. Microbiome Analyses of Blood and Tissues Suggest Cancer Diagnostic Approach. Nature (2020) 579:567-74. doi: 10.1038/s41586-020-2095-1

37. Daillere R, Vetizou M, Waldschmitt N, Yamazaki T, Isnard C, PoirierColame V, et al. Enterococcus Hirae and Barnesiella Intestinihominis Facilitate Cyclophosphamide-Induced Therapeutic Immunomodulatory Effects. Immunity (2016) 45:931-43. doi: 10.1016/j.immuni.2016.09.009

38. Viaud S, Saccheri F, Mignot G, Yamazaki T, Daillère R, Hannani D, et al. The Intestinal Microbiota Modulates the Anticancer Immune Effects of Cyclophosphamide. Sci (New York NY) (2013) 342:971-6. doi: 10.1126/ science. 1240537

39. Nejman D, Livyatan I, Fuks G, Gavert N, Zwang Y, Geller LT, et al. The Human Tumor Microbiome is Composed of Tumor Type-Specific Intracellular Bacteria. Sci (New York NY) (2020) 368:973-80. doi: 10.1126/ science.aay 9189

40. Geller LT, Barzily-Rokni M, Danino T, Jonas OH, Shental N, Nejman D, et al. Potential Role of Intratumor Bacteria in Mediating Tumor Resistance to the Chemotherapeutic Drug Gemcitabine. Sci (New York NY) (2017) 357:1156-60. doi: 10.1126/science.aah5043

41. Sivan A, Corrales L, Hubert N, Williams JB, Aquino-Michaels K, Earley ZM, et al. Commensal Bifidobacterium Promotes Antitumor Immunity and Facilitates Anti-PD-L1 Efficacy. Sci (New York NY) (2015) 350:1084-9. doi: $10.1126 /$ science.aac 4255

42. Thaiss CA, Levy M, Grosheva I, Zheng D, Soffer E, Blacher E, et al. Hyperglycemia Drives Intestinal Barrier Dysfunction and Risk for Enteric Infection. Sci (New York NY) (2018) 359:1376-83. doi: 10.1126/science.aar3318

43. Iida N, Dzutsev A, Stewart CA, Smith L, Bouladoux N, Weingarten RA, et al. Commensal Bacteria Control Cancer Response to Therapy by Modulating the Tumor Microenvironment. Sci (New York NY) (2013) 342:967-70. doi: $10.1126 /$ science. 1240527

44. Feng M, Chen JY, Weissman-Tsukamoto R, Volkmer JP, Ho PY, McKenna KM, et al. Macrophages Eat Cancer Cells Using Their Own Calreticulin as a Guide: Roles of TLR and Btk. Proc Natl Acad Sci USA (2015) 112:2145-50. doi: $10.1073 /$ pnas. 1424907112

45. Tseng D, Volkmer JP, Willingham SB, Contreras-Trujillo H, Fathman JW, Fernhoff NB, et al. Anti-CD47 Antibody-Mediated Phagocytosis of Cancer by Macrophages Primes an Effective Antitumor T-Cell Response. Proc Natl Acad Sci USA (2013) 110:11103-8. doi: 10.1073/pnas. 1305569110

46. Gil-Cruz C, Perez-Shibayama C, De Martin A, Ronchi F, van der Borght K, Niederer R, et al. Microbiota-Derived Peptide Mimics Drive Lethal Inflammatory Cardiomyopathy. Sci (New York NY) (2019) 366:881-6. doi: $10.1126 /$ science.aav3487

47. Ji Q, Perchellet A, Goverman JM. Viral Infection Triggers Central Nervous System Autoimmunity via Activation of CD8 + T Cells Expressing Dual TCRs. Nat Immunol (2010) 11:628-34. doi: 10.1038/ni.1888

48. Bradley CP, Teng F, Felix KM, Sano T, Naskar D, Block KE, et al. Segmented Filamentous Bacteria Provoke Lung Autoimmunity by Inducing Gut-Lung Axis Th17 Cells Expressing Dual TCRs. Cell Host Microbe (2017) 22:697704.e694. doi: 10.1016/j.chom.2017.10.007

49. Balachandran VP, Luksza M, Zhao JN, Makarov V, Moral JA, Remark R, et al. Identification of Unique Neoantigen Qualities in Long-Term Survivors of Pancreatic Cancer. Nature (2017) 551:512-6. doi: 10.1038/nature24462

50. Vujanovic L, Mandic M, Olson WC, Kirkwood JM, Storkus WJ. A Mycoplasma Peptide Elicits Heteroclitic CD4+ T Cell Responses Against Tumor Antigen MAGE-A6. Clin Cancer Res (2007) 13:6796-806. doi: 10.1158/1078-0432.CCR-07-1909

51. Vétizou M, Pitt JM, Daillère R, Lepage $P$, Waldschmitt N, Flament $C$, et al. Anticancer Immunotherapy by CTLA-4 Blockade Relies on the Gut Microbiota. Sci (New York NY) (2015) 350:1079-84. doi: 10.1126/ science.aad1329

52. Bessell CA, Isser A, Havel JJ, Lee S, Bell DR, Hickey JW, et al. Commensal Bacteria Stimulate Antitumor Responses via T Cell Cross-Reactivity. JCI Insight (2020) 5:e135597. doi: 10.1172/jci.insight.135597

53. Fluckiger A, Daillère R, Sassi M, Sixt BS, Liu P, Loos F, et al. Cross-Reactivity Between Tumor MHC Class I-Restricted Antigens and an Enterococcal Bacteriophage. Sci (New York NY) (2020) 369:936-42. doi: 10.1126/ science.aax0701 
54. Nicholson JK, Holmes E, Kinross J, Burcelin R, Gibson G, Jia W, et al. HostGut Microbiota Metabolic Interactions. Sci (New York NY) (2012) 336:12627. doi: $10.1126 /$ science. 1223813

55. Levy M, Blacher E, Elinav E. Microbiome, Metabolites and Host Immunity. Curr Opin Microbiol (2017) 35:8-15. doi: 10.1016/j.mib.2016.10.003

56. Iraporda C, Errea A, Romanin DE, Cayet D, Pereyra E, Pignataro O, et al. Lactate and Short Chain Fatty Acids Produced by Microbial Fermentation Downregulate Proinflammatory Responses in Intestinal Epithelial Cells and Myeloid Cells. Immunobiology (2015) 220:1161-9. doi: 10.1016/ j.imbio.2015.06.004

57. Vinolo MA, Rodrigues HG, Hatanaka E, Sato FT, Sampaio SC, Curi R. Suppressive Effect of Short-Chain Fatty Acids on Production of Proinflammatory Mediators by Neutrophils. J Nutr Biochem (2011) 22:849-55. doi: 10.1016/j.jnutbio.2010.07.009

58. Macia L, Tan J, Vieira AT, Leach K, Stanley D, Luong S, et al. MetaboliteSensing Receptors GPR43 and GPR109A Facilitate Dietary Fibre-Induced Gut Homeostasis Through Regulation of the Inflammasome. Nat Commun (2015) 6:6734. doi: 10.1038/ncomms7734

59. Chang PV, Hao L, Offermanns S, Medzhitov R. The Microbial Metabolite Butyrate Regulates Intestinal Macrophage Function via Histone Deacetylase Inhibition. Proc Natl Acad Sci USA (2014) 111:2247-52. doi: 10.1073/ pnas. 1322269111

60. Gurav A, Sivaprakasam S, Bhutia YD, Boettger T, Singh N, Ganapathy V. Slc5a8, a Na+-Coupled High-Affinity Transporter for Short-Chain Fatty Acids, Is a Conditional Tumour Suppressor in Colon That Protects Against Colitis and Colon Cancer Under Low-Fibre Dietary Conditions. Biochem J (2015) 469:267-78. doi: 10.1042/bj20150242

61. White CA, Pone EJ, Lam T, Tat C, Hayama KL, Li G, et al. Histone Deacetylase Inhibitors Upregulate B Cell microRNAs That Silence AID and Blimp-1 Expression for Epigenetic Modulation of Antibody and Autoantibody Responses. J Immunol (Baltimore Md: 1950) (2014) 193:5933-50. doi: 10.4049/jimmunol.1401702

62. Smith PM, Howitt MR, Panikov N, Michaud M, Gallini CA, Bohlooly YM, et al. The Microbial Metabolites, Short-Chain Fatty Acids, Regulate Colonic Treg Cell Homeostasis. Sci (New York NY) (2013) 341:569-73. doi: 10.1126/ science. 1241165

63. Coutzac C, Jouniaux JM, Paci A, Schmidt J, Mallardo D, Seck A, et al. Systemic Short Chain Fatty Acids Limit Antitumor Effect of CTLA-4 Blockade in Hosts With Cancer. Nat Commun (2020) 11:2168. doi: 10.1038/s41467-020-16079-x

64. Nomura M, Nagatomo R, Doi K, Shimizu J, Baba K, Saito T, et al. Association of Short-Chain Fatty Acids in the Gut Microbiome With Clinical Response to Treatment With Nivolumab or Pembrolizumab in Patients With Solid Cancer Tumors. JAMA Netw Open (2020) 3:e202895. doi: 10.1001/jamanetworkopen.2020.2895

65. Peng Z, Cheng S, Kou Y, Wang Z, Jin R, Hu H, et al. The Gut Microbiome Is Associated With Clinical Response to Anti-PD-1/PD-L1 Immunotherapy in Gastrointestinal Cancer. Cancer Immunol Res (2020) 8:1251-61. doi: 10.1158/2326-6066.CIR-19-1014

66. Renner K, Bruss C, Schnell A, Koehl G, Becker HM, Fante M, et al. Restricting Glycolysis Preserves T Cell Effector Functions and Augments Checkpoint Therapy. Cell Rep (2019) 29:135-50.e139. doi: 10.1016/j.celrep. 2019.08.068

67. Chen J, Cao X, Li B, Zhao Z, Chen S, Lai SWT, et al. Warburg Effect Is a Cancer Immune Evasion Mechanism Against Macrophage Immunosurveillance. Front Immunol (2020) 11:621757. doi: 10.3389/ fimmu.2020.621757

68. Carriche GM, Almeida L, Stuve P, Velasquez L, Dhillon-LaBrooy A, Roy U, et al. Regulating T-Cell Differentiation Through the Polyamine Spermidine. J Allergy Clin Immunol (2021) 147:335-48.e311. doi: 10.1016/j.jaci. 2020.04.037

69. He B, Hoang TK, Wang T, Ferris M, Taylor CM, Tian X, et al. Resetting Microbiota by Lactobacillus Reuteri Inhibits T Reg Deficiency-Induced Autoimmunity via Adenosine A2A Receptors. J Exp Med (2017) 214:10723. doi: $10.1084 / \mathrm{jem} .20160961$

70. Wang T, Gnanaprakasam JNR, Chen X, Kang S, Xu X, Sun H, et al. Inosine Is an Alternative Carbon Source for CD8(+)-T-Cell Function Under Glucose Restriction. Nat Metab (2020) 2:635-47. doi: 10.1038/s42255-020-0219-4
71. Chaput N, Lepage P, Coutzac C, Soularue E, Le Roux K, Monot C, et al. Baseline Gut Microbiota Predicts Clinical Response and Colitis in Metastatic Melanoma Patients Treated With Ipilimumab. Ann Oncol (2017) 28:136879. doi: $10.1093 /$ annonc/mdx108

72. Frankel AE, Coughlin LA, Kim J, Froehlich TW, Xie Y, Frenkel EP, et al. Metagenomic Shotgun Sequencing and Unbiased Metabolomic Profiling Identify Specific Human Gut Microbiota and Metabolites Associated With Immune Checkpoint Therapy Efficacy in Melanoma Patients. Neoplasia (2017) 19:848-55. doi: 10.1016/j.neo.2017.08.004

73. Gopalakrishnan V, Spencer CN, Nezi L, Reuben A, Andrews MC, Karpinets TV, et al. Gut Microbiome Modulates Response to Anti-PD-1 Immunotherapy in Melanoma Patients. Sci (New York NY) (2018) 359:97103. doi: $10.1126 /$ science.aan 4236

74. Matson V, Fessler J, Bao R, Chongsuwat T, Zha Y, Alegre ML, et al. The Commensal Microbiome is Associated With Anti-PD-1 Efficacy in Metastatic Melanoma Patients. Sci (New York NY) (2018) 359:104-8. doi: $10.1126 /$ science.aao3290

75. Wind TT, Gacesa R, Vich Vila A, de Haan JJ, Jalving M, Weersma RK, et al. Gut Microbial Species and Metabolic Pathways Associated With Response to Treatment With Immune Checkpoint Inhibitors in Metastatic Melanoma. Melanoma Res (2020) 30:235-46. doi: 10.1097/CMR.0000000000000656

76. Jin Y, Dong H, Xia L, Yang Y, Zhu Y, Shen Y, et al. The Diversity of Gut Microbiome Is Associated With Favorable Responses to Anti-Programmed Death 1 Immunotherapy in Chinese Patients With NSCLC. J Thorac Oncol (2019) 14:1378-89. doi: 10.1016/j.jtho.2019.04.007

77. Hakozaki T, Richard C, Okuma Y, Derosa L, Elkrief A, Zitvogel L, et al. Gut Microbiome to Predict Efficacy and Immune-Related Toxicities in Patients With Advanced Non-Small Cell Lung Cancer Treated With Anti-PD-1/PDL1 Antibody-Based Immunotherapy. Am Soc Clin Oncol (2020) 38:3095. doi: 10.1200/JCO.2020.38.15_suppl.3095

78. Katayama Y, Yamada T, Shimamoto T, Iwasaku M, Kaneko Y, Uchino J, et al. The Role of the Gut Microbiome on the Efficacy of Immune Checkpoint Inhibitors in Japanese Responder Patients With Advanced non-Small Cell Lung Cancer. Transl Lung Cancer Res (2019) 8:847-53. doi: $10.21037 /$ tlcr.2019.10.23

79. Song P, Yang D, Wang H, Cui X, Si X, Zhang X, et al. Relationship Between Intestinal Flora Structure and Metabolite Analysis and Immunotherapy Efficacy in Chinese NSCLC Patients. Thorac Cancer (2020) 11:1621-32. doi: 10.1111/1759-7714.13442

80. Fukuoka S, Daisuke M, Togashi Y, Sugiyama E, Udagawa H, Kirita K, et al. Association of Gut Microbiome With Immune Status and Clinical Response in Solid Tumor Patients Who Received on Anti-PD-1 Therapies. Am Soc Clin Oncol (2018) 36:3011. doi: 10.1200/JCO.2018.36.15_suppl.3011

81. Routy B, Le Chatelier E, Derosa L, Duong CPM, Alou MT, Daillère R, et al. Gut Microbiome Influences Efficacy of PD-1-Based Immunotherapy Against Epithelial Tumors. Sci (New York NY) (2018) 359:91-7. doi: 10.1126/ science.aan 3706

82. Agarwal A, Modliszewski J, Davey L, Reyes-Martinez M, Runyambo D, Corcoran D, et al. Investigating the Role of the Gastrointestinal Microbiome in Response to Immune Checkpoint Inhibitors (ICIs) Among Patients (Pts) With Metastatic Renal Cell Carcinoma (mRCC). Am Soc Clin Oncol (2020) 38:730. doi: 10.1200/JCO.2020.38.6_suppl.730

83. Heshiki Y, Vazquez-Uribe R, Li J, Ni Y, Quainoo S, Imamovic L, et al. Predictable Modulation of Cancer Treatment Outcomes by the Gut Microbiota. Microbiome (2020) 8:28. doi: 10.1186/s40168-020-00811-2

84. Yin H, Yang L, Peng G, Yang K, Mi Y, Hu X, et al. The Commensal Consortium of the Gut Microbiome Is Associated With Favorable Responses to Anti-Programmed Death Protein 1 (PD-1) Therapy in Thoracic Neoplasms. Cancer Biol Med (2021) 18. doi: 10.20892/j.issn.20953941.2020 .0450

85. Zheng Y, Wang T, Tu X, Huang Y, Zhang H, Tan D, et al. Gut Microbiome Affects the Response to Anti-PD-1 Immunotherapy in Patients With Hepatocellular Carcinoma. J Immunother Cancer (2019) 7:193. doi: 10.1186/s40425-019-0650-9

86. Liu D, Schilling B, Liu D, Sucker A, Livingstone E, Jerby-Arnon L, et al. Integrative Molecular and Clinical Modeling of Clinical Outcomes to PD1 Blockade in Patients With Metastatic Melanoma. Nat Med (2019) 25:191627. doi: 10.1038/s41591-019-0654-5 
87. Batten M, Shanahan E, Simpson R, Read M, Silva IP, Angelatos A, et al. Gut Microbiota Predicts Response and Toxicity With Neoadjuvant Immunotherapy. AACR (2020) 80:5734. doi: 10.1158/1538-7445.AM2020-5734

88. Raggi D, Bandini M, Pederzoli F, Giannatempo P, Marandino L, Basile G, et al. Concomitant Antibiotics (ATBs) Use and Survival Outcomes in Patients (Pts) With Muscle-Invasive Bladder Cancer (MIBC) Treated With Neoadjuvant Pembrolizumab (PURE-01 Study). Am Soc Clin Oncol (2021) 39:449. doi: 10.1200/JCO.2021.39.6_suppl.449

89. Tanoue T, Morita S, Plichta DR, Skelly AN, Suda W, Sugiura Y, et al. A Defined Commensal Consortium Elicits CD8 T Cells and Anti-Cancer Immunity. Nature (2019) 565:600-5. doi: 10.1038/s41586-019-0878-Z

90. Roberti MP, Yonekura S, Duong CPM, Picard M, Ferrere G, Tidjani Alou M, et al. Chemotherapy-Induced Ileal Crypt Apoptosis and the Ileal Microbiome Shape Immunosurveillance and Prognosis of Proximal Colon Cancer. Nat Med (2020) 26:919-31. doi: 10.1038/s41591-020-0882-8

91. Ivanov II, Atarashi K, Manel N, Brodie EL, Shima T, Karaoz U, et al. Induction of Intestinal Th17 Cells by Segmented Filamentous Bacteria. Cell (2009) 139:485-98. doi: 10.1016/j.cell.2009.09.033

92. Xu X, Lv J, Guo F, Li J, Jia Y, Jiang D, et al. Gut Microbiome Influences the Efficacy of PD-1 Antibody Immunotherapy on MSS-Type Colorectal Cancer via Metabolic Pathway. Front Microbiol (2020) 11:814. doi: 10.3389/ fmicb.2020.00814

93. Feng M, Jiang W, Kim BYS, Zhang CC, Fu Y-X, Weissman IL. Phagocytosis Checkpoints as New Targets for Cancer Immunotherapy. Nat Rev Cancer (2019) 19:568. doi: 10.1038/s41568-019-0183-z

94. Derosa L, Routy B, Fidelle M, Iebba V, Alla L, Pasolli E, et al. Gut Bacteria Composition Drives Primary Resistance to Cancer Immunotherapy in Renal Cell Carcinoma Patients. Eur Urol (2020) 78:195-206. doi: 10.1016/ j.eururo.2020.04.044

95. Sistigu A, Viaud S, Chaput N, Bracci L, Proietti E, Zitvogel L. Immunomodulatory Effects of Cyclophosphamide and Implementations for Vaccine Design. Semin Immunopathol (2011) 33:369-83. doi: 10.1007/ s00281-011-0245-0

96. Schiavoni G, Sistigu A, Valentini M, Mattei F, Sestili P, Spadaro F, et al. Cyclophosphamide Synergizes With Type I Interferons Through Systemic Dendritic Cell Reactivation and Induction of Immunogenic Tumor Apoptosis. Cancer Res (2011) 71:768-78. doi: 10.1158/0008-5472.can-102788

97. Ghiringhelli F, Larmonier N, Schmitt E, Parcellier A, Cathelin D, Garrido C, et al. CD4+CD25+ Regulatory T Cells Suppress Tumor Immunity But are Sensitive to Cyclophosphamide Which Allows Immunotherapy of Established Tumors to be Curative. Eur J Immunol (2004) 34:336-44. doi: 10.1002/eji.200324181

98. Viaud S, Flament C, Zoubir M, Pautier P, LeCesne A, Ribrag V, et al. Cyclophosphamide Induces Differentiation of Th17 Cells in Cancer Patients. Cancer Res (2011) 71:661-5. doi: 10.1158/0008-5472.can-10-1259

99. Baruch EN, Youngster I, Ben-Betzalel G, Ortenberg R, Lahat A, Katz L, et al. Fecal Microbiota Transplant Promotes Response in ImmunotherapyRefractory Melanoma Patients. Sci (New York NY) (2020) 371:602-9. doi: 10.1126/science.abb5920

100. Davar D, Dzutsev AK, McCulloch JA, Rodrigues RR, Chauvin J-M, Morrison RM, et al. Fecal Microbiota Transplant Overcomes Resistance to Anti-PD-1 Therapy in Melanoma Patients. Sci (New York NY) (2021) 371:595-602. doi: $10.1126 /$ science.abf 3363

101. Maleki S, Lenehan J, Burton J, Silverman M, Parvathy SN, El-Hajjar M, et al. P864 Combination of Fecal Microbiota Transplantation From Healthy Donors With Anti-PD1 Immunotherapy in Treatment-Naïve Advanced or Metastatic Melanoma Patients. BMJ Specialist J (2020) 8:A11-2. doi: 10.1136/LBA2019.17

102. Cammarota G, Ianiro G, Gasbarrini A. Fecal Microbiota Transplantation for the Treatment of Clostridium Difficile Infection: A Systematic Review. J Clin Gastroenterol (2014) 48:693-702. doi: 10.1097/mcg.0000000000000046

103. Alang N, Kelly CR. Weight Gain After Fecal Microbiota Transplantation. Open Forum Infect Dis (2015) 2:ofv004. doi: 10.1093/ofid/ofv004

104. Zhernakova A, Kurilshikov A, Bonder MJ, Tigchelaar EF, Schirmer M, Vatanen T, et al. Population-Based Metagenomics Analysis Reveals Markers for Gut Microbiome Composition and Diversity. Sci (New York NY) (2016) 352:565-9. doi: 10.1126/science.aad3369
105. Falony G, Joossens M, Vieira-Silva S, Wang J, Darzi Y, Faust K, et al. Population-Level Analysis of Gut Microbiome Variation. Sci (New York NY) (2016) 352:560-4. doi: 10.1126/science.aad3503

106. Claesson MJ, Jeffery IB, Conde S, Power SE, O'Connor EM, Cusack S, et al. Gut Microbiota Composition Correlates With Diet and Health in the Elderly. Nature (2012) 488:178-84. doi: 10.1038/nature11319

107. Muegge BD, Kuczynski J, Knights D, Clemente JC, González A, Fontana L, et al. Diet Drives Convergence in Gut Microbiome Functions Across Mammalian Phylogeny and Within Humans. Sci (New York NY) (2011) 332:970-4. doi: 10.1126/science.1198719

108. Rajilić-Stojanović M, Heilig HG, Tims S, Zoetendal EG, de Vos WM. LongTerm Monitoring of the Human Intestinal Microbiota Composition. Environ Microbiol (2012) 15:1146-59. doi: 10.1111/1462-2920.12023

109. Makki K, Deehan EC, Walter J, Backhed F. The Impact of Dietary Fiber on Gut Microbiota in Host Health and Disease. Cell Host Microbe (2018) 23:705-15. doi: 10.1016/j.chom.2018.05.012

110. Hryckowian AJ, Van Treuren W, Smits SA, Davis NM, Gardner JO, Bouley DM, et al. Microbiota-Accessible Carbohydrates Suppress Clostridium Difficile Infection in a Murine Model. Nat Microbiol (2018) 3:662-9. doi: 10.1038/s41564-018-0150-6

111. Kamada N, Kim YG, Sham HP, Vallance BA, Puente JL, Martens EC, et al. Regulated Virulence Controls the Ability of a Pathogen to Compete With the Gut Microbiota. Sci (New York NY) (2012) 336:1325-9. doi: 10.1126/ science. 1222195

112. Ramirez-Farias C, Slezak K, Fuller Z, Duncan A, Holtrop G, Louis P. Effect of Inulin on the Human Gut Microbiota: Stimulation of Bifidobacterium Adolescentis and Faecalibacterium Prausnitzii. Br J Nutr (2009) 101:54150. doi: 10.1017/s0007114508019880

113. Rangan P, Choi I, Wei M, Navarrete G, Guen E, Brandhorst S, et al. FastingMimicking Diet Modulates Microbiota and Promotes Intestinal Regeneration to Reduce Inflammatory Bowel Disease Pathology. Cell Rep (2019) 26:2704-19.e2706. doi: 10.1016/j.celrep.2019.02.019

114. Di Biase S, Lee C, Brandhorst S, Manes B, Buono R, Cheng CW, et al. Fasting-Mimicking Diet Reduces HO-1 to Promote T Cell-Mediated Tumor Cytotoxicity. Cancer Cell (2016) 30:136-46. doi: 10.1016/j.ccell.2016.06.005

115. Wu GD, Chen J, Hoffmann C, Bittinger K, Chen YY, Keilbaugh SA, et al. Linking Long-Term Dietary Patterns With Gut Microbial Enterotypes. Sci (New York NY) (2011) 334:105-8. doi: 10.1126/science.1208344

116. Laute-Caly DL, Raftis EJ, Cowie P, Hennessy E, Holt A, Panzica DA, et al. The Flagellin of Candidate Live Biotherapeutic Enterococcus Gallinarum MRx0518 is a Potent Immunostimulant. Sci Rep (2019) 9:801. doi: 10.1038/ s41598-018-36926-8

117. Drake CG, Pachynski RK, Subudhi SK, McNeel DG, Antonarakis ES, Bauer TM, et al. Safety and Preliminary Immunogenicity of JNJ-64041809, a Live Attenuated, Double-Deleted Listeria Monocytogenes-Based Immunotherapy, in Metastatic Castration-Resistant Prostate Cancer (mCRPC). J Clin Oncol (2019) 37:e16509-9. doi: 10.1200/JCO.2019.37.15_suppl.e16509

118. Chowdhury S, Castro S, Coker C, Hinchliffe TE, Arpaia N, Danino T. Programmable Bacteria Induce Durable Tumor Regression and Systemic Antitumor Immunity. Nat Med (2019) 25:1057-63. doi: 10.1038/s41591019-0498-z

119. Gurbatri CR, Lia I, Vincent R, Coker C, Castro S, Treuting PM, et al. Engineered Probiotics for Local Tumor Delivery of Checkpoint Blockade Nanobodies. Sci Trans Med (2020) 12:eaax0876. doi: 10.1126/ scitranslmed.aax0876

120. Leventhal DS, Sokolovska A, Li N, Plescia C, Kolodziej SA, Gallant CW, et al. Immunotherapy With Engineered Bacteria by Targeting the STING Pathway for Anti-Tumor Immunity. Nat Commun (2020) 11:2739. doi: 10.1038/ s41467-020-16602-0

121. Uribe-Herranz M, Rafail S, Beghi S, Gil-de-Gomez L, Verginadis I, Bittinger K, et al. Gut Microbiota Modulate Dendritic Cell Antigen Presentation and Radiotherapy-Induced Antitumor Immune Response. J Clin Invest (2020) 130:466-79. doi: 10.1172/JCI124332

122. Uribe-Herranz M, Bittinger K, Rafail S, Guedan S, Pierini S, Tanes C, et al. Gut Microbiota Modulates Adoptive Cell Therapy via CD8alpha Dendritic Cells and IL-12. JCI Insight (2018) 3:e94952. doi: 10.1172/jci.insight.94952

123. Elkrief A, El Raichani L, Richard C, Messaoudene M, Belkaid W, Malo J, et al. Antibiotics are Associated With Decreased Progression-Free Survival of 
Advanced Melanoma Patients Treated With Immune Checkpoint Inhibitors. Oncoimmunology (2019) 8:e1568812. doi: 10.1080/2162402X.2019.1568812

124. Derosa L, Hellmann MD, Spaziano M, Halpenny D, Fidelle M, Rizvi H, et al. Negative Association of Antibiotics on Clinical Activity of Immune Checkpoint Inhibitors in Patients With Advanced Renal Cell and nonSmall-Cell Lung Cancer. Ann Oncol (2018) 29:1437-44. doi: 10.1093/ annonc/mdy103

125. Zhao S, Gao G, Li W, Li X, Zhao C, Jiang T, et al. Antibiotics are Associated With Attenuated Efficacy of Anti-PD-1/PD-L1 Therapies in Chinese Patients With Advanced non-Small Cell Lung Cancer. Lung Cancer (2019) 130:10-7. doi: 10.1016/j.lungcan.2019.01.017

126. Hakozaki T, Richard C, Elkrief A, Hosomi Y, Benlaifaoui M, Mimpen I, et al. The Gut Microbiome Associates With Immune Checkpoint Inhibition Outcomes in Patients With Advanced Non-Small Cell Lung Cancer. Cancer Immunol Res (2020) 8:1243-50. doi: 10.1158/23266066.CIR-20-0196

127. Pinato DJ, Howlett S, Ottaviani D, Urus H, Patel A, Mineo T, et al. Association of Prior Antibiotic Treatment With Survival and Response to Immune Checkpoint Inhibitor Therapy in Patients With Cancer. JAMA Oncol (2019) 5:1774-8. doi: 10.1001/jamaoncol.2019.2785

128. Lalani AA, Xie W, Braun DA, Kaymakcalan M, Bosse D, Steinharter JA, et al. Effect of Antibiotic Use on Outcomes With Systemic Therapies in Metastatic Renal Cell Carcinoma. Eur Urol Oncol (2020) 3:372-81. doi: 10.1016/ j.euo.2019.09.001

129. Skelly AN, Sato Y, Kearney S, Honda K. Mining the Microbiota for Microbial and Metabolite-Based Immunotherapies. Nat Rev Immunol (2019) 19:30523. doi: 10.1038/s41577-019-0144-5

130. Nam GH, Hong Y, Choi Y, Kim GB, Kim YK, Yang Y, et al. An Optimized Protocol to Determine the Engulfment of Cancer Cells by Phagocytes Using Flow Cytometry and Fluorescence Microscopy. J Immunol Methods (2019) 470:27-32. doi: 10.1016/j.jim.2019.04.007

131. Poh CM, Zheng J, Channappanavar R, Chang ZW, Nguyen THO, Rénia L, et al. Multiplex Screening Assay for Identifying Cytotoxic CD8(+) T Cell Epitopes. Front Immunol (2020) 11:400. doi: 10.3389/fimmu.2020.00400

132. Stein RR, Tanoue T, Szabady RL, Bhattarai SK, Olle B, Norman JM, et al. Computer-Guided Design of Optimal Microbial Consortia for Immune System Modulation. eLife (2018) 7:e30916. doi: 10.7554/eLife.30916
133. Marcos-Zambrano LJ, Karaduzovic-Hadziabdic K, Loncar Turukalo T, Przymus P, Trajkovik V, Aasmets O, et al. Applications of Machine Learning in Human Microbiome Studies: A Review on Feature Selection, Biomarker Identification, Disease Prediction and Treatment. Front Microbiol (2021) 12:634511. doi: 10.3389/fmicb.2021.634511

134. Maldonado-Gómez MX, Martínez I, Bottacini F, O'Callaghan A, Ventura M, van Sinderen D, et al. Stable Engraftment of Bifidobacterium Longum AH1206 in the Human Gut Depends on Individualized Features of the Resident Microbiome. Cell Host Microbe (2016) 20:515-26. doi: 10.1016/ j.chom.2016.09.001

135. Shepherd ES, DeLoache WC, Pruss KM, Whitaker WR, Sonnenburg JL. An Exclusive Metabolic Niche Enables Strain Engraftment in the Gut Microbiota. Nature (2018) 557:434-8. doi: 10.1038/s41586-018-0092-4

136. Litvak Y, Byndloss MX, Bäumler AJ. Colonocyte Metabolism Shapes the Gut Microbiota. Sci (New York NY) (2018) 362:eaat9076. doi: 10.1126/ science.aat 9076

137. Zheng D, Liwinski T, Elinav E. Interaction Between Microbiota and Immunity in Health and Disease. Cell Res (2020) 30:492-506. doi: $10.1038 / \mathrm{s} 41422-020-0332-7$

Conflict of Interest: The authors declare that the research was conducted in the absence of any commercial or financial relationships that could be construed as a potential conflict of interest.

Publisher's Note: All claims expressed in this article are solely those of the authors and do not necessarily represent those of their affiliated organizations, or those of the publisher, the editors and the reviewers. Any product that may be evaluated in this article, or claim that may be made by its manufacturer, is not guaranteed or endorsed by the publisher.

Copyright $\odot 2021 \mathrm{Li}$, Gong, Hao, Zhou and Cheng. This is an open-access article distributed under the terms of the Creative Commons Attribution License (CC BY). The use, distribution or reproduction in other forums is permitted, provided the original author(s) and the copyright owner(s) are credited and that the original publication in this journal is cited, in accordance with accepted academic practice. No use, distribution or reproduction is permitted which does not comply with these terms. 